\title{
On the Measurement of Angular Dependent Sound Transmission through Airborne Supercritical Plates
}

\author{
Matthew D. Shaw \\ Brigham Young University - Provo
}

Follow this and additional works at: https://scholarsarchive.byu.edu/etd

Part of the Astrophysics and Astronomy Commons, and the Physics Commons

\section{BYU ScholarsArchive Citation}

Shaw, Matthew D., "On the Measurement of Angular Dependent Sound Transmission through Airborne Supercritical Plates" (2011). Theses and Dissertations. 2835.

https://scholarsarchive.byu.edu/etd/2835

This Thesis is brought to you for free and open access by BYU ScholarsArchive. It has been accepted for inclusion in Theses and Dissertations by an authorized administrator of BYU ScholarsArchive. For more information, please contact scholarsarchive@byu.edu, ellen_amatangelo@byu.edu. 
On the Measurement of Airborne, Angular-dependent Sound Transmission through Supercritical Bars

\author{
Matthew D. Shaw
}

A thesis submitted to the faculty of

Brigham Young University

in partial fulfillment of the requirements for the degree of

Master of Science

Kent L. Gee, Advisor

Scott D. Sommerfeldt

Karl F. Warnick

Brian E. Anderson

Department of Physics and Astronomy

Brigham Young University

December 2011

Copyright ( 2011 Matthew D. Shaw

All Rights Reserved 


\author{
ABSTRACT \\ On the Measurement of Airborne, Angular-dependent Sound Transmission \\ through Supercritical Bars \\ Matthew D. Shaw \\ Department of Physics and Astronomy, BYU \\ Master of Science
}

\begin{abstract}
A method of measuring angular dependence of acoustic transmission through supercritical plates in air is discussed. The coincidence effect occurs in a supercritical plate when the component of the acoustic wave number parallel to the plate matches the bending wave number in the plate. The transmission of sound is a maximum at the angle where this trace wave number matching occurs. The theory of the coincidence effect is well-defined for unbounded thin plates using plane-wave excitation. However, experimental results for finite plates are known to diverge from theory, especially near grazing angles. An experimental setup has been developed in order to observe the coincidence effect using continuous-wave excitation and phased-array methods. Experimental results through a $0.5 \mathrm{~mm}$ thick aluminum bar exhibit strong maxima at the predicted coincidence angles, showing that coincidence is observable using continuous waves. Also, transmission near grazing angles is seen to diverge from infinite plate theory. Further work is suggested to improve the measurement setup and explore the source of the divergence.
\end{abstract}

Keywords: acoustics, sound transmission, coincidence effect, angular dependence, critical frequency 


\section{ACKNOWLEDGMENTS}

Many people have been very influential during the process of this research, and many others have contributed to my success here at BYU. I would like to take this chance to thank a few specific individuals.

First of all, I would like to thank Dr. Brian Anderson for the opportunity to work on this project. He has been a great mentor and friend, as well as a great example of ambition and ability in research. I appreciate that he has showed me how to be a better researcher and professional more than he has just told me. I would also like to thank Drs. Kent Gee, Scott Sommerfeldt, and Karl Warnick, for being willing to advise me on this project. Their experience, knowledge, and understanding have been great resources for me as a student. I would like to thank Dr. Leishman for convincing me to study acoustics and for his time and effort in teaching and mentoring students. Many of my fellow students also deserve my thanks, including Alan Wall, David Krueger, Jarom Giraud, Jesse Daily, and James Esplin, for their friendship and support through these years at BYU. I want to thank Michael Muhlestein for his help in designing the experimental setup, Wesley Lifferth for building it, and Blaine Harker for taking measurements regarding the extraction of material properties.

Last of all, I want to thank all of my family for their love and unfailing support. My siblings have been good friends during my time as a student. My mother has always been a source of strength for me. My father is an excellent example of an eternal student, and I thank him for teaching me the value of education and doing a good job. Most importantly, I want to thank my wonderful wife, Charlyn, for her patience and kind heart. I will always appreciate the comfort that she has brought me. 


\section{Table of Contents}

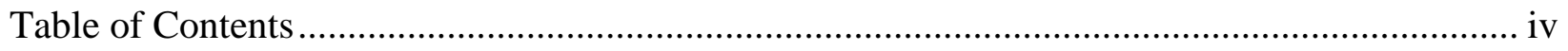

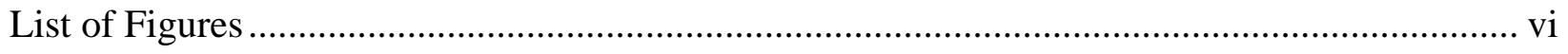

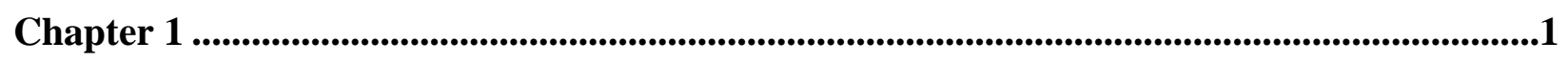

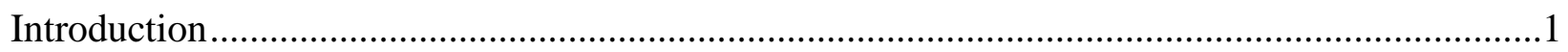

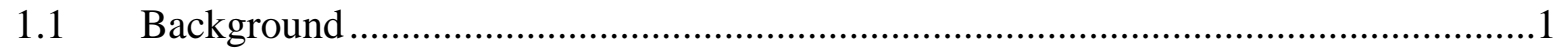

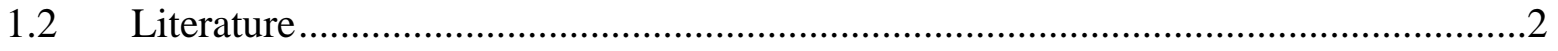

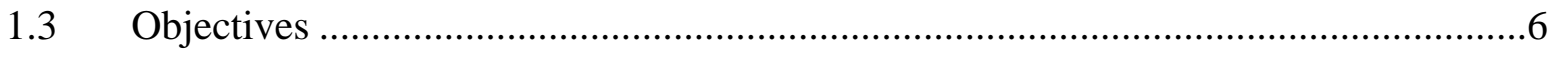

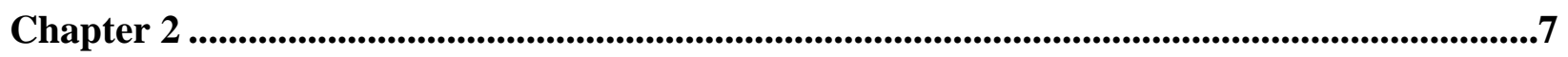

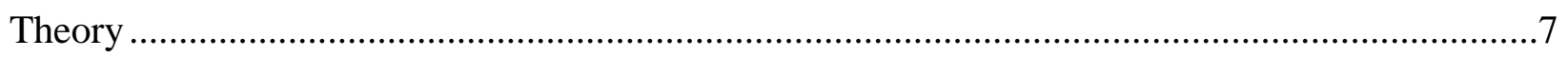

Chapter 3 ................................................................................................................................11

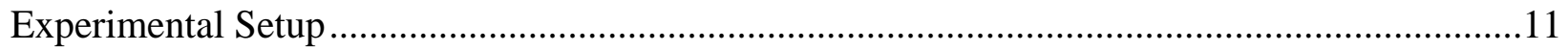

3.1 Equipment and Physical Setup................................................................... 11

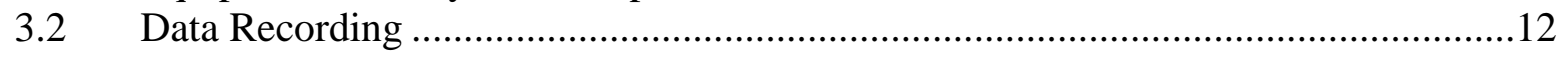

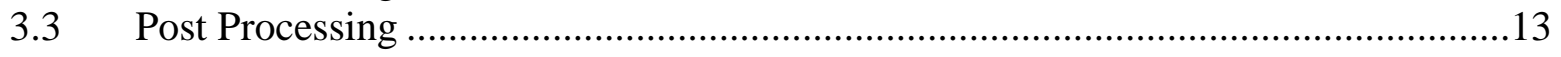

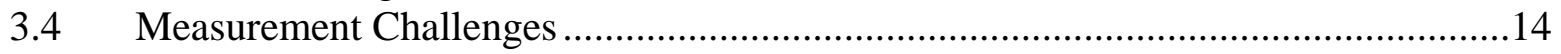

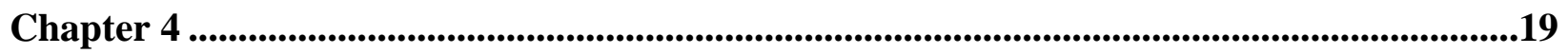

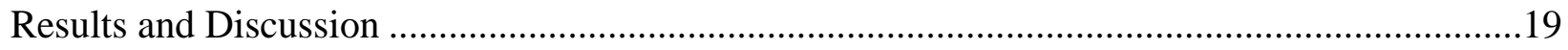

Chapter 5 .........................................................................................................................................................27

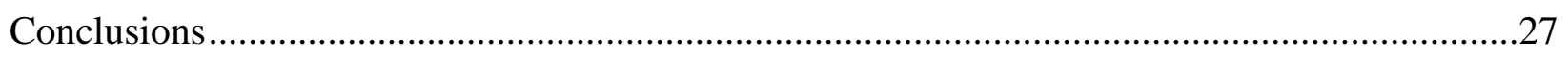

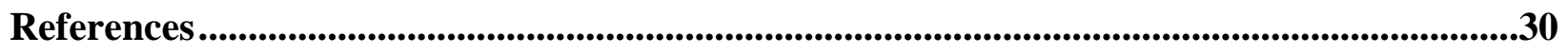

Appendix A

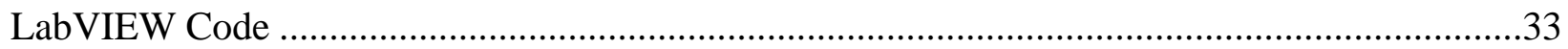

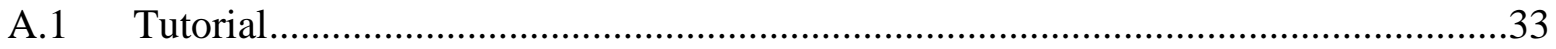

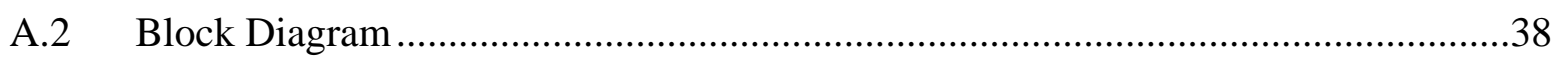

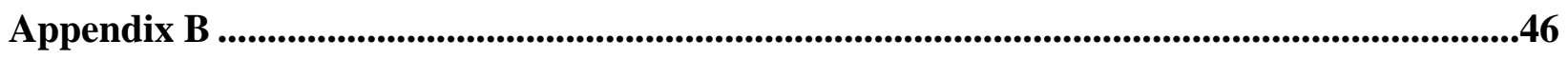




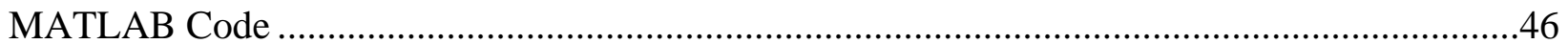

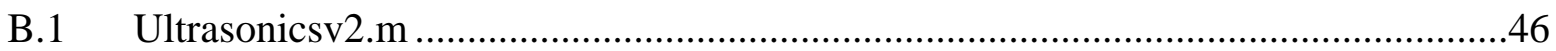

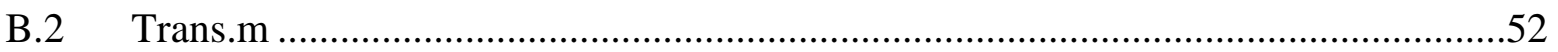

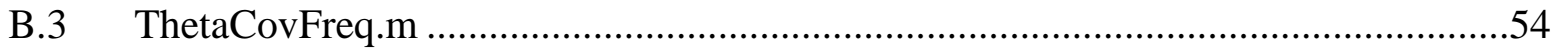

Appendix C

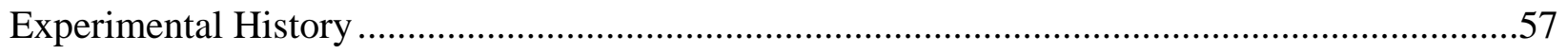

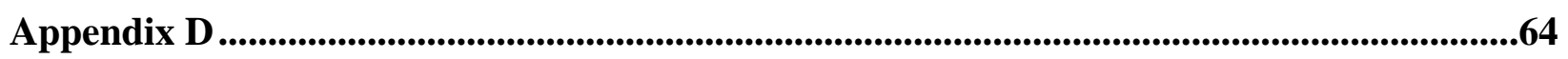

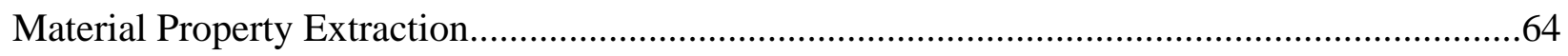




\section{List of Figures}

FIG. 2.1. Plot of the theoretical angular-dependent transmission through a $0.5-\mathrm{mm}$ aluminum plate at a subcritical and at a supercritical frequency. The normal-incidence mass law for both frequencies is given for comparison. ....................................................................... 9

FIG. 3.1. Photographs of the experimental setup (a) without the bar with the $65 \mathrm{kHz}$ source and

(b) with the bar, foam baffle, and $45 \mathrm{kHz}$ source. 18

FIG. 4.1. Five sequential blocks recorded by microphone 4 at an incident angle of $47.5^{\circ}$ from normal. (a) Portion showing the arrival of the acoustic wave during one of the blocks. (b) Zoomed in portion showing the repeatability of five blocks.

FIG. 4.2. Steered data with and without the bar for (a) $0^{\circ}$ and (b) $47.5^{\circ}$ incidence. The difference between the markers is the transmission at the respective angle. 22

FIG. 4.3. Transmission through the 0.5 -mm aluminum bar versus angle at $45 \mathrm{kHz} \ldots \ldots \ldots \ldots \ldots \ldots \ldots . . .23$

FIG. 4.4. Comparison of five transmission measurements made without removing the bar........ 24

FIG. 4.5. Comparison of five transmission measurements made while removing and reattaching the bar. 25

FIG. 4.6. Transmission through the $0.5 \mathrm{~mm}$ aluminum bar versus angle at $65 \mathrm{kHz}$................... 26

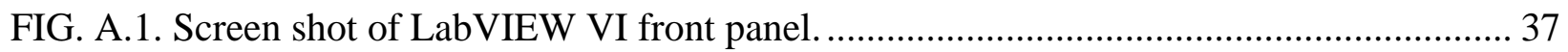


FIG. A.2. Screen shot of the entire LabVIEW VI block diagram.

FIG. A.3. The turntable initialization section of the VI block diagram...................................... 41

FIG. A.4. The while loop in the VI block diagram that waits for the user to begin the

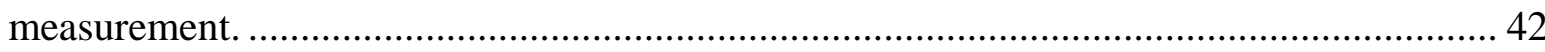

FIG. A.5. DAQ initialization in the VI block diagram. ............................................................ 43

FIG. A.6. Measurement log file compilation in the VI block diagram......................................... 44

FIG. A.7. The first section of the measurement loop of the VI block diagram. ........................... 45

FIG. A.8. The second part of the measurement loop in the VI block diagram............................. 46

FIG. A.9. The final section of the measurement loop in the VI block diagram. .......................... 47

FIG. C.1. Results of transmission during early experiments at $40.5 \mathrm{kHz}$ using foam that was not

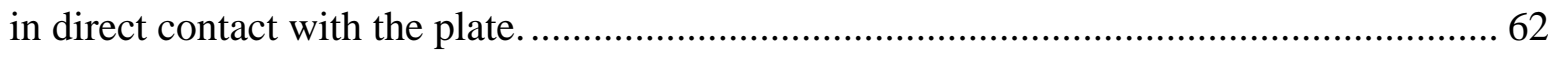

FIG. C.2. Scanning laser vibrometer measurements of a small section of the large plate. (a) $0^{\circ}$

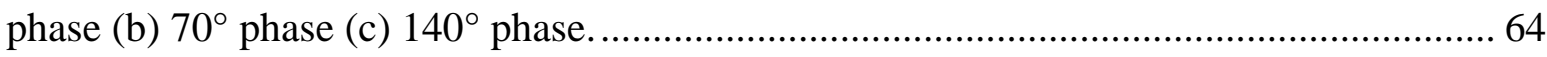

FIG. D.1. Surface plot of the transmission for frequency versus angle with color representing the transmission levels for the aluminum bar. .......................................................................... 71

FIG. D.2. Coincidence angle versus frequency for the aluminum bar. ..................................... 71 


\section{Chapter 1}

\section{Introduction}

\subsection{Background}

Sound transmission loss through barriers has been studied in depth for various purposes, including noise control and architectural acoustics. Many studies have aimed at determining the transmission loss of barriers as a function of frequency at normal incidence or at an oblique angle of incidence. The angular dependence of sound transmission at a specific frequency has also been studied and defined theoretically. Knowing this dependence has proven useful in various applications, including using supercritical plates as angular filters for sound transmission. ${ }^{1}$

There exists a critical frequency for a given plate, which is when the wave speed in the surrounding fluid matches the bending wave speed in the plate. At this frequency, an incident acoustic wave at grazing incidence $\left( \pm 90^{\circ}\right.$ from normal incidence) excites a traveling bending wave in the plate, which then radiates at grazing incidence on the transmitted side of the plate, 
with a maximum sound transmission, compared to other angles of incidence. Above the critical frequency (supercritical region), the bending wave speed exceeds the speed of sound in the fluid, and there exists an angle at which the component of the acoustic wave number parallel to the plate matches the bending wave number in the plate (this occurs at both positive and negative mirrored angles about normal incidence). At this angle, called the coincidence angle, the incident acoustic wave excites a traveling flexural wave in the plate at the bending wave speed. The coincidence effect results in maximum sound transmission at the coincidence angle (compared to angles below and above the coincidence angle), as the traveling bending wave radiates acoustically on the transmitted side of the plate. Since the bending wave speed is dispersive for thin plates, this angle shifts towards normal incidence as frequency increases. Additionally, if an acoustic wave is incident upon the plate at an angle between grazing incidence and the coincidence angle, then a traveling wave is still excited in the plate but at a speed less than the bending wave speed. Conversely, if an acoustic wave is incident at an angle between the coincidence angle and normal incidence then a traveling wave at a speed greater than the bending wave speed is excited in the plate. Acoustic radiation from the plate, and hence transmission through the plate, is most efficient when the wave traveling in the plate matches the bending wave speed.

\subsection{Literature}

The development of the theory of angular-dependent sound transmission of plane waves through unbounded, flexible, thin plates is credited to Cremer in $1942 .^{2}$ It was later confirmed 
numerically by Lamb in $1956^{3}$ and experimentally by Eichler and Lambert in $1957 .{ }^{4}$ Since then, there have been many studies to better understand the coincidence effect and its applications.

In 1971, Bhattacharya et al. ${ }^{5}$ found that for a finite plate, it was impossible to observe the coincidence effect in steady state without backing the plate with a cavity. This conclusion was based on previous work by Bhattacharya and Crocker ${ }^{6}$ where they found that for a plate backed by a cavity, the coincidence effect could be observed through matching of flexural, standing waves in the plate and standing waves in the acoustic cavity. This resulted in a coincidence effect independent of angle, and it could only be observed at a critical frequency and subsequent discrete coincidence frequencies that corresponded with cavity modes. They made this distinction their plate was finite and asserted that coincidence could not be observed in finite plates in the same manner that it can be as Cremer had proposed for infinite plates. They claimed that freely propagating waves could not exist in a finite plate. In their measurement setup, they essentially measured better transmission when the cavity or the plate was excited at a resonance. However, standing waves in one dimension fundamentally consist of two traveling waves. Thus traveling waves can be excited in a finite plate and possibly result in coincidence if an individual traveling wave may be monitored. One of the major shortcomings of their experimental setup was the use of a single microphone inside the cavity which prevented them from directly sensing angular dependent sound transmission.

Ten years later, Davies and Gibbs ${ }^{7}$ suggested that the conclusions of Bhattacharya et al. were valid only for steady state conditions and proposed exciting the finite plate using shortduration source signals. Their work was based on the approach used by Raes ${ }^{8}$ and Louden, ${ }^{9}$ in which an impulse was measured before and after transmitting through an impedance change (the 
end of a pipe or a partition, for example), and the comparison of the amplitudes of the two spectra yielded the transmission properties. One purpose of using short-duration source signals was the ease of separating in time any diffracted pulses from the transmitted pulse. Thus large plates were required for their technique. The waveform they used was a $50 \mu$ s square pulse. While its duration is short enough to allow separation of the transmitted pulse from diffracted pulses, its energy is spread over a wide frequency range and therefore the signal-to-noise ratio (SNR) is poor relative to monochromatic excitation.

Anderson et al. $^{10}$ used the pulse approach for finite length bars submerged in water, except that they used a time-windowed, narrow band pulse rather than an impulse or a square wave pulse. ${ }^{10}$ This yielded good signal amplitude at a specific frequency (the center frequency of the pulse). For their experiment, several transducers in a line array were mounted to an alumina bar, and the combination was rotated to various angles as sound energy impinged on the bar. They did not have to separate the transmitted arrival of sound from diffracted arrivals because their array was surrounded by air, aside from the faces of the transducers being mounted to the bar to detect the transmitted sound. An acoustic window kept the cavity surrounding the array from being filled with water. Diffracted waves would be greatly reduced in amplitude due to the impedance mismatch from the acoustic window to the air and then again from the air to the transducers. The transducers recorded the transmitted sound at each rotation angle. Using phase delays in post processing, the main lobe of the array directivity pattern was steered towards the source for each rotation angle, allowing the receiving array to be most sensitive in the direction of transmission. A comparison of transmitted amplitudes to those collected by an array without the alumina bar gave the transmission of the bar as a function of incident angle. The advantage of 
their work is that smaller plates and bars could be studied, since the array provided better sensitivity in the direction of the transmitted sound and due to the reduced diffraction.

While the results of Anderson et al. matched theory for infinite plates at most angles of incidence, they noticed that towards grazing incidence, the theory for infinite plates and experimental results from finite bars began to diverge. Theory predicts perfect transmission at grazing angles irrespective of material properties of the plate. The results of Anderson et al. at grazing angles were corrupted by measurement noise floors and thus the degree of departure from theory could not be quantified with certainty. Furthermore, when calculating the diffuse field transmission loss of a finite partition, Fahy and Gardonio ${ }^{11}$ omit angles greater than $78^{\circ}$ in their integration. By doing so, they found that the calculated values better match those collected from experiments. Others suggest that this is "fortuitous" and not grounded scientifically and propose other methods to correct for discrepancies. ${ }^{12-14}$ Despite this argument, there still remains a discrepancy between experiment and theory towards grazing angles.

The literature shows a lack of experimental data that exhibit the coincidence effect using continuous waves. One purpose of using continuous waves is to obtain a better SNR at a chosen frequency. The literature also shows a lack of experimental validation of the divergence of theory from experiment at grazing angles. Better experimental results confirming this discrepancy could increase understanding of the physical phenomena, and understanding the degree of departure between theory and experiment could potentially lead to developing more appropriate theoretical equations for finite plates. 


\subsection{Objectives}

The purpose of this thesis is to describe a measurement system similar to that of Anderson et al. to investigate the angular dependence of airborne sound transmission through finite supercritical bars using continuous waves, i.e. in steady state. This thesis will show that it is possible to observe the coincidence effect using continuous waves without backing the bar with a cavity. It also provides further evidence of the departure of theory from experiment near grazing angles.

In this thesis, a summary of the theory of oblique-incidence sound transmission through unbounded plates will be given. The experimental setup used to measure supercritical sound transmission through finite plates will then be discussed. Challenges related to the experiments will also be addressed. Results obtained from experiments will be shown and discussed. Finally, conclusions will be made, and possible further research will be suggested. 


\section{Chapter 2}

\section{Theory}

This chapter presents a summary for the theory of the coincidence effect in thin, flexible plates of infinite extent. A more complete derivation of oblique-incidence sound transmission is given elsewhere. ${ }^{11,15}$ This theory provides a good foundation for the angular-dependent transmission through finite plates.

The transmission coefficient as a function of incident angle, $\tau(\theta)$, for a single-frequency plane wave incident on an unbounded thin plate is governed by

$$
\tau(\theta)=\frac{\left(2 \rho_{0} c \sec \theta\right)^{2}}{\left[2 \rho_{0} c \sec \theta+(D / \omega) \eta k^{4} \sin ^{4} \theta\right]^{2}+\left[\omega m-(D / \omega) k^{4} \sin ^{4} \theta\right]^{2}}
$$

The numerator and the first term in the denominator represent the sum of the fluid loading on the transmitted and incident sides, dependent on the density of the fluid, $\rho_{0}$, the speed of sound in the fluid, $c$, and the angle of incidence, $\theta$, measured from the normal to the surface of the plate. 
The second term in the denominator is governed by the damping in the plate, with dependence on the loss factor, $\eta$, of the plate, the bending stiffness of the plate, $D$, the angular frequency, $\omega$, and the acoustic wave number, $k$. The third and fourth terms are governed by the mass of the plate and the stiffness of the plate, respectively. The mass per unit area, $m$, and the bending stiffness are defined as

$$
m=\rho h,
$$

and

$$
D=\frac{E h^{3}}{12\left(1-\sigma^{2}\right)}
$$

respectively, where $\rho$ is the density of the plate material, $h$ is the thickness of the plate, $E$ is Young's modulus, and $\sigma$ is Poisson's ratio. It is often useful to plot the transmission loss (TL) as the logarithm of the inverse of the transmission coefficient. Here we deem it more appropriate to plot the transmission, $T(\theta)$, which is calculated as

$$
T(\theta)=10 \log _{10} \tau(\theta)
$$

Plots of Eq. (2.4) are given in Fig. 2.1 for a $0.5 \mathrm{~mm}$ thick aluminum plate at a subcritical frequency $(500 \mathrm{~Hz})$ and at a supercritical frequency $(45 \mathrm{kHz})$. (For aluminum, $\rho$ is $2700 \mathrm{~kg} / \mathrm{m}^{3}$, $E$ is $70 \mathrm{GPa}, \sigma$ is 0.33 , and $\eta$ is $1 \times 10^{-5}$.) The normal incidence mass law transmission (transmission as opposed to transmission loss), 


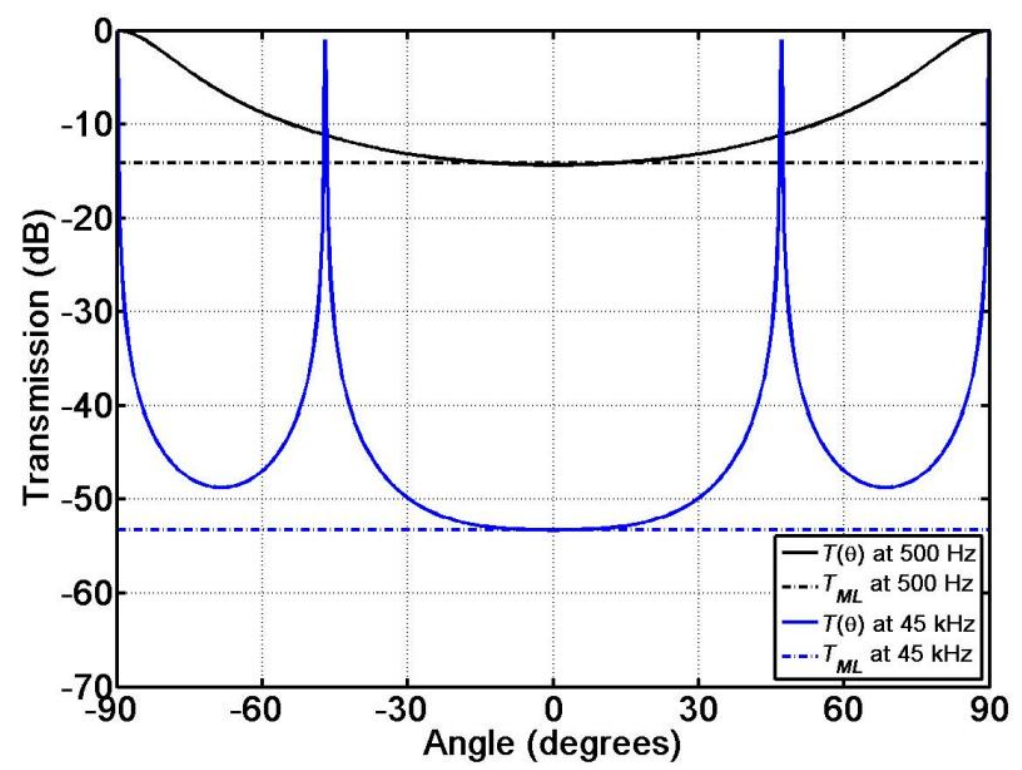

FIG. 2.1. Plot of the theoretical angular-dependent transmission through a 0.5-mm aluminum plate at a subcritical and at a supercritical frequency. The normal-incidence mass law for both frequencies is given for comparison.

$$
T_{M L}=-10 \log _{10}\left[\frac{m \omega}{2 \rho_{0} c}\right]^{2}
$$

is also included for comparison (note its absence of angular dependence). The subcritical frequency curve shows the absence of coincidence peaks, while the supercritical frequency curve contains two sharp peaks at the coincidence angles around $\pm 47^{\circ}$. These peaks graphically illustrate the coincidence effect, which occurs when the mass and bending stiffness terms in the denominator of Eq. (2.1) cancel each other, thus maximizing $T$ (away from grazing incidence). The coincidence angle, $\theta_{C O}$, for a specific frequency can therefore be calculated by equating these two terms as 


$$
\sin ^{2} \theta_{C O}=\frac{c^{2}}{\omega} \sqrt{\frac{m}{D}}=\frac{c^{2}}{c_{B}^{2}}
$$

where

$$
c_{B}=\left(\omega^{2} \frac{D}{m}\right)^{1 / 4}
$$

is the (dispersive) bending wave speed in the plate. It must be noted that for subcritical frequencies, the bending wave speed is less than the acoustic wave speed, and coincidence is not observed.

The critical frequency, $f_{c}$, can be calculated by solving Eq. (2.6) when $\theta_{c o}$ is $90^{\circ}$, yielding

$$
f_{c}=\frac{c^{2}}{2 \pi} \sqrt{\frac{m}{D}}
$$

For aluminum, with a thickness of $0.5 \mathrm{~mm}$, the critical frequency is $24.1 \mathrm{kHz}$. As frequency increases above the critical frequency, the coincidence angle decreases from grazing angles toward normal incidence with increasing frequency. Further manipulation of Eq. (2.6) yields the mathematical result described above that the component of the acoustic wave number parallel to the plate matches the bending wave number:

$$
c_{B}^{2} \sin ^{2} \theta_{C O}=c^{2} \Rightarrow k_{B}=k \sin \theta_{C O}
$$

(Figure 5.3 of Reference 11 gives a visualization of this wave number matching on p. 288.) 


\section{Chapter 3}

\section{Experimental Setup}

The purpose of this section is to explain the experimental setup used to obtain the results presented in Chapter 4. A more detailed explanation of the history regarding the development of this experimental setup is given in Appendix C.

\subsection{Equipment and Physical Setup}

The angular dependent transmission measurements described below were taken inside an anechoic chamber. ${ }^{16} \mathrm{~A}$ turntable capable of rotating at $2.5^{\circ}$ increments was controlled using LabVIEW. (See Appendix A for more information about the LabVIEW program used for these measurements.) With the same LabVIEW program, data were streamed at $204.8 \mathrm{kHz}$ per channel through National Instruments PXI-4461 and 4462 cards from eight $6.35 \mathrm{~mm}(0.25$ inch) GRAS type-I pre-polarized microphones. These microphones were placed in a line array mounted on the 
turntable with center-to-center spacing of $6.35 \mathrm{~mm}$ between array elements, such that the midpoint of the array at the microphone diaphragms lie on the axis of rotation.

A stationary ultrasonic source was pointed towards the center of the microphone array and synchronized to the microphone input channels. For measurements at $45 \mathrm{kHz}$, a Parasonics Corp. 4012A ultrasonic transducer (center frequency of $40 \mathrm{kHz}$ ) was used. For measurements at $65 \mathrm{kHz}$, a Parasonics Corp. PAR58T ultrasonic transducer (center frequency of $58 \mathrm{kHz}$ ) was used. The corresponding single-frequency sine wave was created in LabVIEW with an amplitude of $5 \mathrm{~V}$ and sent through the PXI to control the source. The signal was also routed back into an input and recorded in order to compute transfer functions between the source signal and the microphone recordings in post processing.

\subsection{Data Recording}

To begin a measurement the turntable was rotated to $-90^{\circ}$ away from normal incidence to the array, and five 16384-point data blocks were recorded. For each block, the source signal was a single-frequency sine wave that started and stopped in the 16384 point data set. The turntable was then rotated $2.5^{\circ}$ towards normal incidence, and five more blocks were recorded. This same operation was performed at each $2.5^{\circ}$ increment, through normal incidence, to $+90^{\circ}$. In order to obtain a transmission measurement, the entire process was repeated with an aluminum bar in front of the array, and the without-bar and with-bar measurements were compared.

For each microphone, at each rotation angle, the five recorded blocks of data were analyzed with MATLAB (see Appendix B for the MATLAB code). The transient part at the beginning of each time signal, including the time of flight and transient portion of the sound 
from the source, was removed in order to ensure analysis of only the steady state portion of the microphone signals. The resultant blocks were then windowed using a Hanning window. In order to reduce the effect of noise in the signals and to average out variations in magnitude and phase between blocks, the windowed blocks were summed together in the time domain. This synchronous averaging is analogous to complex vector averaging done in frequency domain. A fast Fourier transform (FFT) of each summed microphone signal was taken, and the (complex) value at the source frequency was extracted. The transfer function, $H_{n}$, between this value and the corresponding value from the source signal was calculated as

$$
H_{n}=\frac{a^{*} b_{n}}{a^{*} a}
$$

where $a$ is the value of the FFT from the source signal, $b_{n}$ is the value from the $n$-th microphone channel, and $*$ denotes a complex conjugate. The magnitude and phase of $H_{n}$ were used in the beam forming process for the $n$-th microphone at each angle. Performing these operations for each rotation angle gave the magnitude and phase of each microphone as a function of rotation angle.

\subsection{Post Processing}

The extracted magnitudes and phases were then added together to compute the directivity of the array, $D_{f, \theta_{0}}(\theta)$, as a function of rotation angle, $\theta$, using the equation

$$
D_{f, \theta_{0}}(\theta)=\sum_{n} A_{f, \theta_{0}, n} e^{j \phi_{f, \theta_{0}, n}} e^{j \omega \delta_{n}}
$$


where $A_{f, \theta_{0}, n}$ is the extracted magnitude from the FFT of the $n$-th microphone signal, $\phi_{f, \theta_{0}, n}$ is the extracted phase, and

$$
\delta_{n}=n d \sin \theta_{0} / c
$$

is the time delay associated with steering the array of spacing, $d$, to a steering angle of $\theta_{0}$. This equation is associated with standard beam forming. ${ }^{17-21}$

When $\theta_{0}$ is set equal to the rotation angle of the turntable, the main lobe of the array is directed towards the source. This was done so that the array was most sensitive to the direct transmission path rather than other paths (to avoid diffraction and grating lobes in the plane of the array). Further, beam forming allowed for separation of the two traveling waves which make up the standing wave in the bar that results from steady-state excitation. The amplitude of the steered pattern was extracted at the steer angle (the main lobe peak of the array) for both the with-bar and without-bar cases, and the logarithm of the ratio of the two was recorded as the transmission at that rotation angle. The transmission as a function of angle was compiled by calculating this ratio for each rotation angle.

\subsection{Measurement Challenges}

Several variables were important to the measurement setup. First, the distance of the source to the bar could determine the sphericity of the wave incident on the bar. Second, the size of the bar could regulate the amount of diffraction that reached the microphones. Third, the boundary conditions of the bar could alter its vibrations and, therefore, its transmission 
properties. Fourth, the distance between the microphone array and the bar could affect the diffraction, the quality of the array beam forming, and, therefore, the transmission measurement.

One experimental difficulty is in creating incident plane waves on the bar. This difficulty was termed the precursor phenomenon by Balilah and Gibbs, ${ }^{22}$ when a spherical wave incident upon a bar excites a spread of flexural wave numbers (instead of a single wave number when using a plane wave), thus allowing for radiation from various angles to corrupt the transmission at the rotated angle. Balilah and Gibbs proposed using larger plates and tedious setup variations to reduce the effect of precursor waves. The beam forming of the array and smaller bar size reduce this effect by being sensitive to a limited spread of wave numbers, as does increasing the distance between the source and the bar. For the experimental setup described here, the ultrasonic sources were placed $90 \mathrm{~cm}(k r=742$ at $45 \mathrm{kHz}$ and $r=0.9 \mathrm{~m}>a=0.02 \mathrm{~m}$, where $r$ is the distance between the source and the center of the array and $a$ is the radius of the source) from the center of the array. This meant that the bar and microphone array were in the acoustic and geometric far field of the source $(k r \gg 1$ and $r \gg a$, respectively), providing a more locally planar wave incident on the bar and reducing the effect of the precursor phenomenon.

Another difficulty is diffraction around the bar. It is possible for diffracted waves to interfere with the transmitted wave, thus corrupting the data. While the use of beam forming can help reduce the sensitivity to diffracted waves in the plane of the array, it is still necessary to attenuate them using a baffle or blocker of some kind, especially for diffraction paths not in the plane of the array. For the case without the bar, the path from the source to the microphones was unimpeded. For the with-bar case, the bar was mounted to a $53 \mathrm{~cm}$ by $46 \mathrm{~cm}$ by $9 \mathrm{~cm}$ foam baffle using duct tape so that the portion exposed to the array was $2 \mathrm{~cm}$ by $13 \mathrm{~cm}$. This baffle supported 
the bar in the desired location and was found to significantly reduce diffraction, while still allowing incident sound energy to transmit through the bar (see Appendix C).

In modal solutions of plates and cavities, boundary conditions play an important role in amplitudes and phases of traveling waves and their reflections. The theory described in Chapter 2 does not include the effects of boundary conditions because of the infinite plate assumption. The boundaries of the bar in this setup do cause reflected waves traveling in the opposite direction which radiate acoustically at the mirrored incident angle about $0^{\circ}$. However, because the array is steered to the incident angle, the effect of the reflected wave should be reduced sufficiently. The effect of the duct tape on the amplitude and reflection of traveling waves in the exposed portion of the bar is unknown but it likely increases the effective damping loss factor for the bar. Further investigations into other methods of securing the bar and reducing diffraction would be necessary to fully understand the influence of boundary conditions on measuring the transmission through bars.

The last experimental parameter, considered here, that could have an impact on results is the distance between the microphones and the bar. Having a bar in the near field of a microphone array could affect the beam forming performed by the array; if the effective media on each side of the bar are different then refraction may occur. Also, diffraction levels become greater, relative to the transmitted sound due to shallower angles at the bar edges, as the array moves farther from the bar. Fortunately, Anderson et al. ${ }^{10}$ found that it was still possible to steer a beam from an array of transducers through a supercritical bar in the near field. They suggested that the array standoff distance be small. The chosen standoff distance here was $5 \mathrm{~mm}$. Further 
investigations could also determine the influence of standoff distance on the experimental transmission results.

For the results given in the next chapter, a $3.8 \mathrm{~cm}$ by $40.6 \mathrm{~cm}$ aluminum bar with a thickness of $0.5 \mathrm{~mm}$, was used. This bar width was determined through experimentation in order to significantly reduce the waves traveling in the direction perpendicular to the array. A larger plate appears to suffer from severe interference fluctuations likely due to traveling waves in the perpendicular direction to the array. See Appendix C. A picture of the final experimental setup, without the bar and with the bar, showing the source, bar, and foam baffle in the anechoic chamber, is given in Fig. 3.1. 


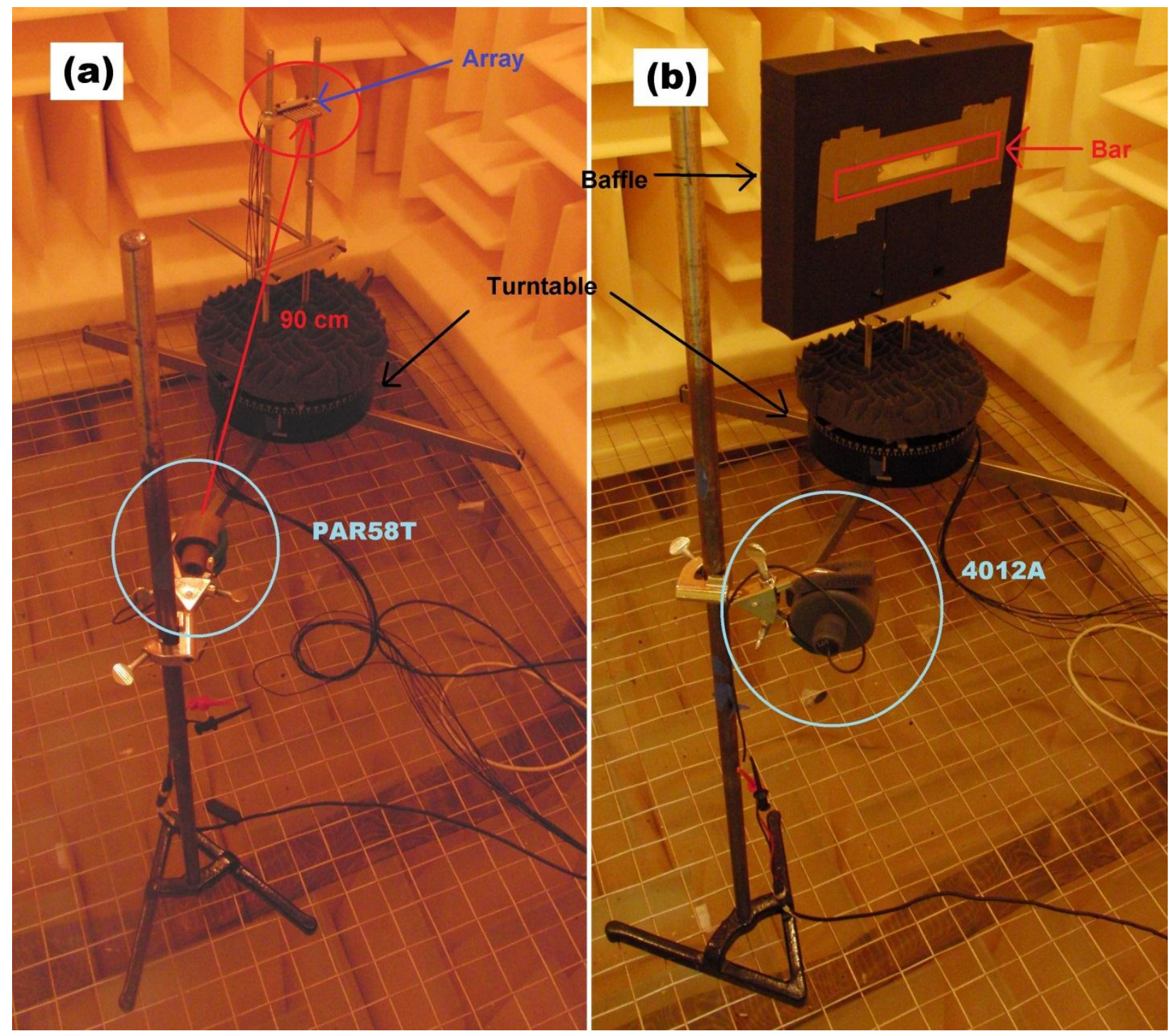

FIG. 3.1. Photographs of the experimental setup (a) without the bar with the $65 \mathrm{kHz}$ source and (b) with the bar, foam baffle, and $45 \mathrm{kHz}$ source. 


\section{Chapter 4}

\section{Results and Discussion}

The intermediate steps of processing the data will be given in this chapter. Data for transmission as a function of angle will be shown, and important results and observations will be discussed.

Figure 4.1 shows portions of the five blocks recorded at the fourth microphone in the array at a rotation angle of $47.5^{\circ}$ from normal incidence. These were chosen as representative waveforms during the experiments. The beginning of the waveform in Fig. 4.1(a) includes the time of flight from the source to the microphones, then a transient portion of the recorded signal, followed by the steady-state portion. The first two portions were removed in the MATLAB processing to analyze the steady-state response of the system only. In Fig. 4.1(b), five blocks are shown to illustrate that the phases of the waveforms are very similar, with deviations in phase corresponding to a single time sample. Variations in phase or amplitude were averaged by summing the five blocks for every microphone and rotation angle. 

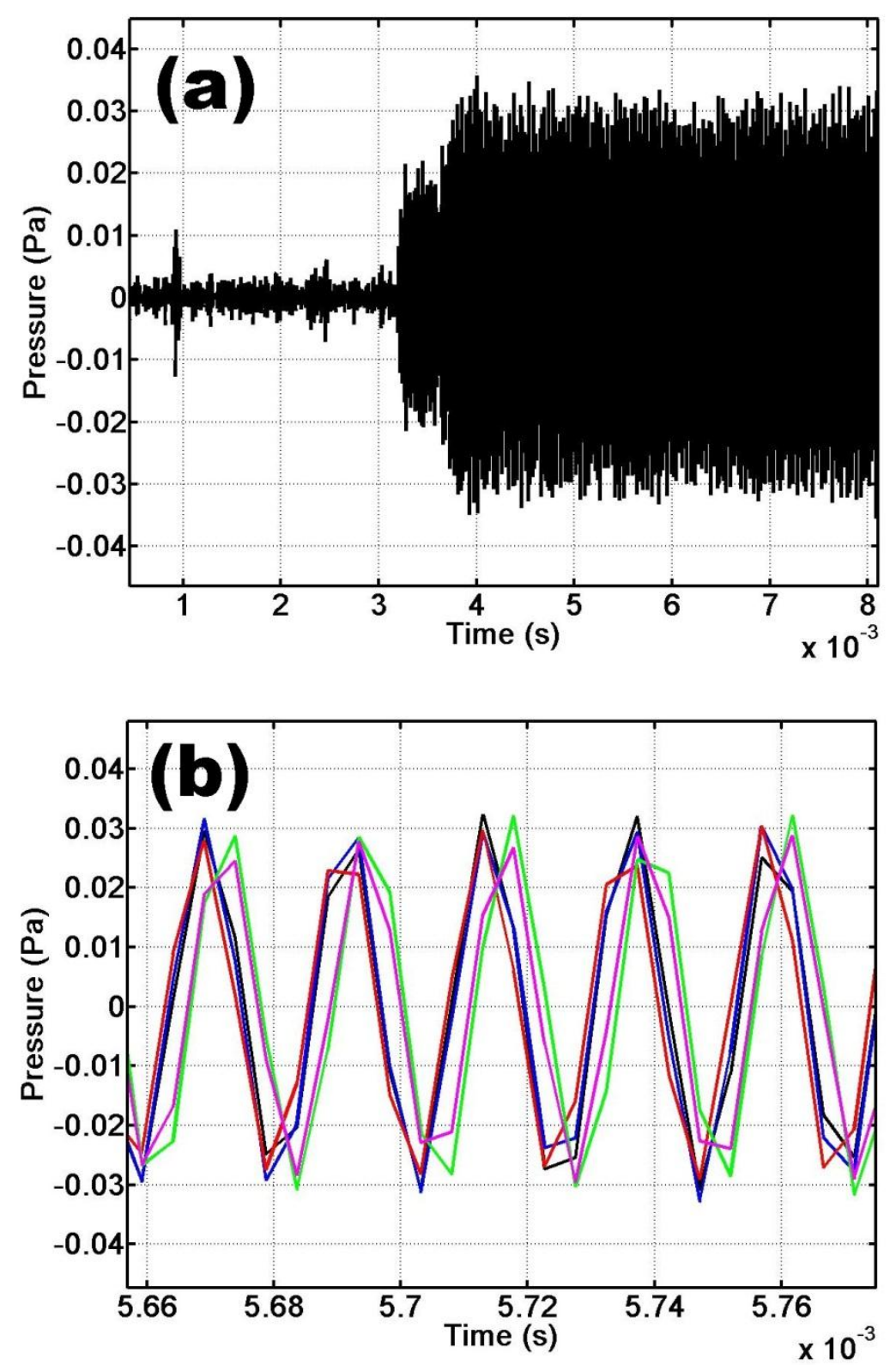

FIG. 4.1. Five sequential blocks recorded by microphone 4 at an incident angle of $47.5^{\circ}$ from normal. (a) Portion showing the arrival of the acoustic wave during one of the blocks. (b) Zoomed in portion showing the repeatability of five blocks. 
The beam forming was performed as described in Eqs. (3.2) and (3.3). Figure 4.2 shows the steered directivities from the array with and without the bar in place. For Fig. 4.2(a), the source was at normal incidence, and the array was steered to $0^{\circ}$. The without-bar curve shows the array directivity pattern. The with-bar curve shows evidence of beam forming through the bar at $0^{\circ}$. It also shows radiation of the bar near the coincidence angles, but the only extracted value from this curve is at $0^{\circ}$. In practice only the values at the main lobes need be calculated. The directivities are shown in Fig. 4.2 for illustrative purposes. The difference between the markers of $54 \mathrm{~dB}$ is the transmission at normal incidence. Figure 4.2(b) shows the data at atation angle of $47.5^{\circ}$, steered to the same angle (near the coincidence angle), and the $30 \mathrm{~dB}$ difference between the markers is the transmission at that angle. The difference is much less at the coincidence angle than at normal incidence, indicating an increase in transmission, as expected. The difference was calculated for each rotation angle, with the array steered towards that angle, and the transmission as a function of angle was therefore determined.

A measurement was taken to determine whether the results were contaminated by the noise floor. The noise floor of the entire system was $9 \mathrm{~dB}$ on average, and the main lobe of the without-bar, steered array directivities was above $90 \mathrm{~dB}$ for each rotation angle. This suggests that the measurement system is capable of measuring transmission levels down to approximately $-81 \mathrm{~dB}$. 

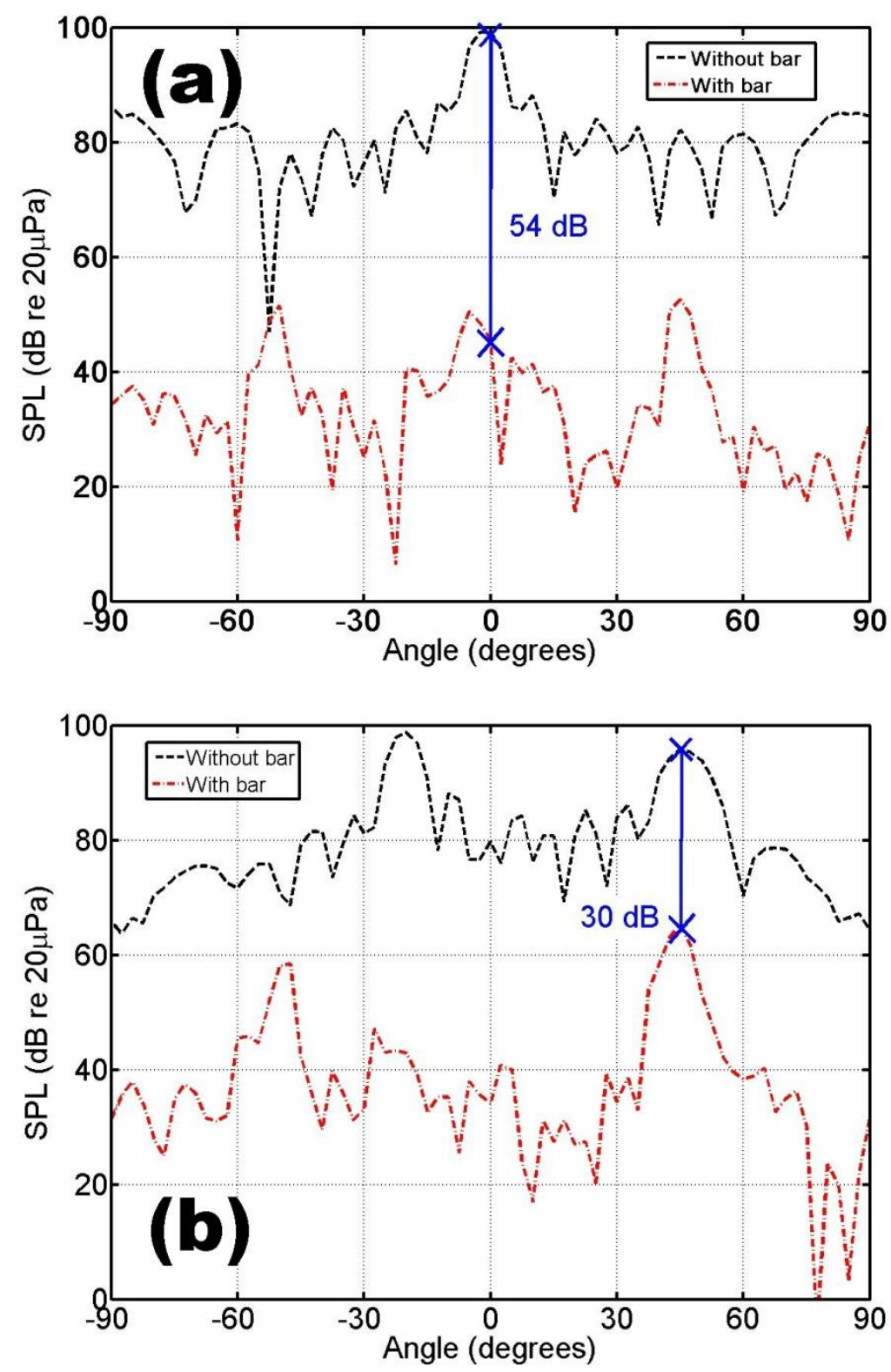

FIG. 4.2. Steered data with and without the bar for (a) $0^{\circ}$ and (b) $47.5^{\circ}$ incidence. The difference between the markers is the transmission at the respective angle.

Figure 4.3 shows the comparison of the theoretical transmission curve, based on Eq. (2.1), and experimental results of the extracted main lobe values as a function of rotation angle. The experimental data show coincidence near the angles predicted from the theoretical equation. 
It must be noted that the coincidence peaks are both shifted in the direction of decreasing angle, indicating that the source placement may have been off by a few degrees. The peak levels are 32 and $26 \mathrm{~dB}$ below the theoretical peak values. The main cause of this is likely poor angular resolution, meaning that the coincidence peaks do not line up exactly with a measurement point. Another cause of this reduction could be small non-uniformities in the bar, preventing pure bending waves from being excited. The spherical wave fronts could also excite a spread of excited flexural wave numbers in the bar, thus rounding and reducing the coincidence peaks. Further, the duct tape may be effectively increasing the damping loss factor in the bar.

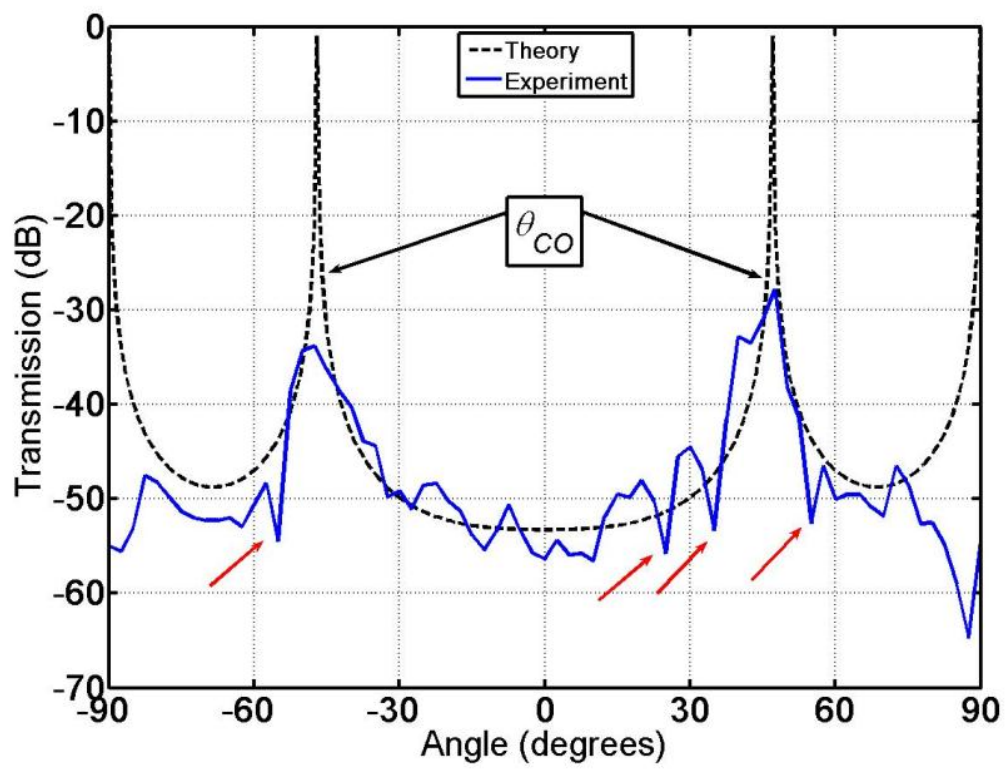

FIG. 4.3. Transmission through the $0.5-\mathrm{mm}$ aluminum bar versus angle at $45 \mathrm{kHz}$.

The data also show agreement with a root mean square error of $2.8 \mathrm{~dB}$ in the mass law region (between $-30^{\circ}$ and $+30^{\circ}$ ). The transmission at grazing incidence is an average of $54.7 \mathrm{~dB}$ less than the perfect transmission predicted by theory. 
The larger fluctuations in the experimental results (indicated in Fig. 4.3 with the red arrows) could have various origins. First, diffraction around the bar might not have been completely reduced by the foam baffle, allowing for interference to corrupt the measurement results. Second, the precursor phenomenon could have affected the measurement in other ways.

In order to further explore the fluctuations, five transmission measurements were taken and compared. For the results shown in Fig. 4.4, the aluminum bar remained attached to the foam baffle. The curves exhibit similar behavior for all angles of incidence, with an average standard deviation of $1.22 \mathrm{~dB}$ (standard deviation was calculated from the five measurements at each angle and then averaged over all rotation angles). This result means that the locations of the fluctuations are not a result of noise floor problems. The cause of these dips, however, is still unknown.

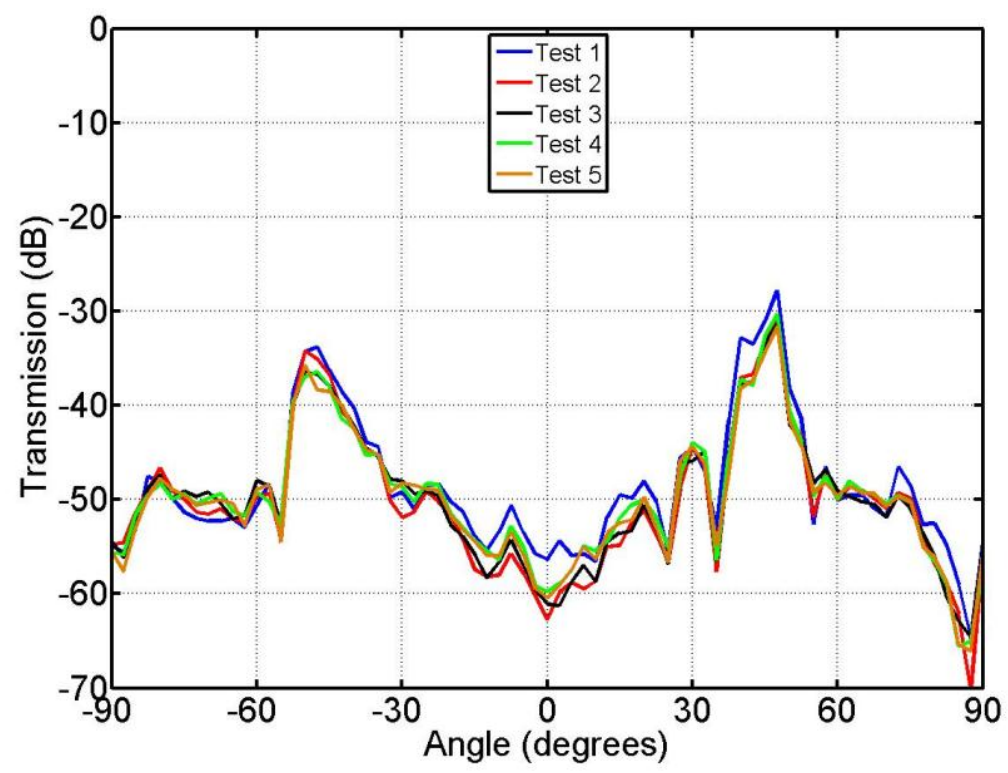

FIG. 4.4. Comparison of five transmission measurements made without removing the bar. 
Another set of five measurements was taken, between which the aluminum bar was removed from the baffle and reattached in the same manner. The results of these five tests are shown in Fig. 4.5. The average standard deviation of these five results is $3.9 \mathrm{~dB}$, more than double that of the curves in Fig. 4.4. The location and amplitude of the fluctuations vary between tests, suggesting that the cause of the fluctuations is related to the bar and how it is mounted to the foam baffle. However, the actual origin of the fluctuations, whether they're due to diffraction or the precursor phenomenon or something else, cannot be determined from these results.

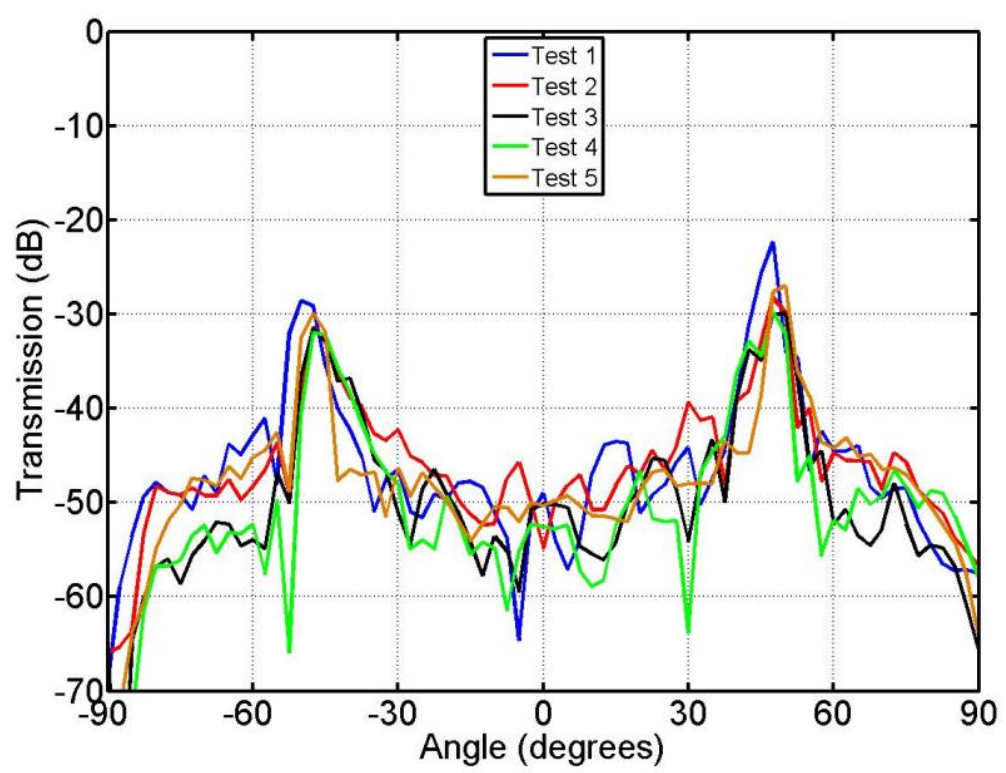

FIG. 4.5. Comparison of five transmission measurements made while removing and reattaching the bar.

Figure 4.6 shows the results for the same experiment at $65 \mathrm{kHz}$. Once again, coincidence is clearly observed at the correct angles. Through this measurement and ones at other frequencies, we have thus found that coincidence does not occur only at specific frequencies for continuous wave excitation of finite bars not back by a cavity, contrary to the findings of Bhattacharya et al..$^{5}$ The peaks are reduced by 26 and $21 \mathrm{~dB}$, possibly a result again of poor 
angular resolution. The root mean square error in the mass law region is $5.7 \mathrm{~dB}$, and the average reduction at grazing angles is $54 \mathrm{~dB}$.

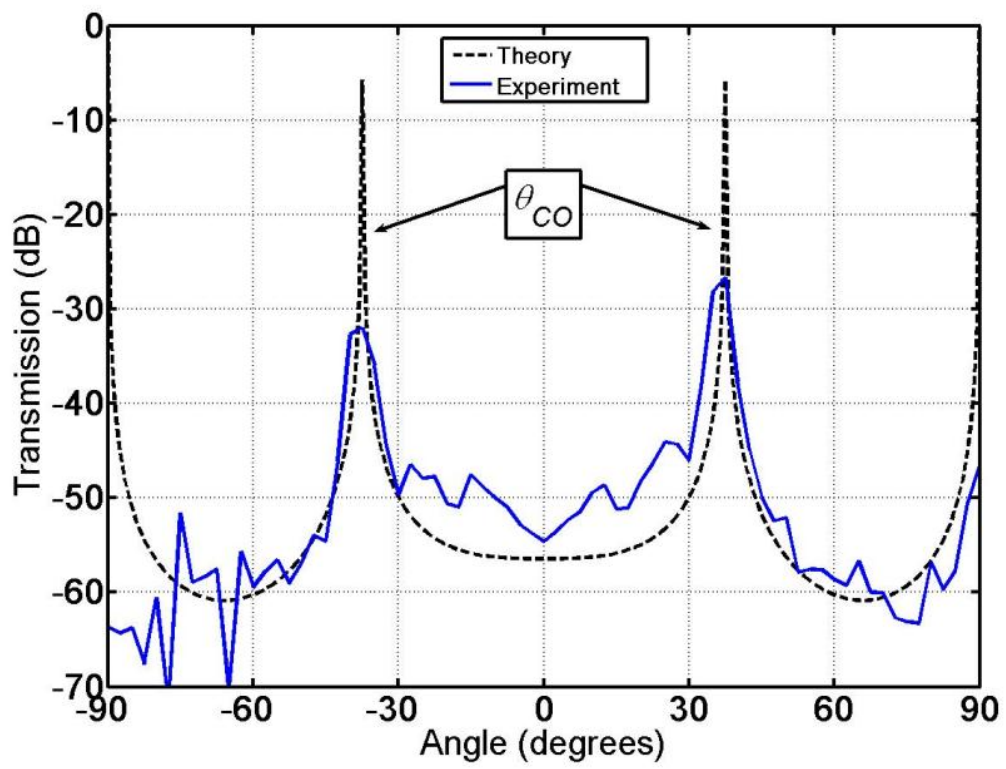

FIG. 4.6. Transmission through the $0.5 \mathrm{~mm}$ aluminum bar versus angle at $65 \mathrm{kHz}$.

The deviations seen in the mass law region of Figs. 4.3 and 4.6 are similar to the deviations seen by Farley and Anderson. ${ }^{23}$ They found a typical insertion loss departure from theory at normal incidence of $5.7 \mathrm{~dB}$ in their experiments on panels of plastic, wood, and steel. The $2.8 \mathrm{~dB}$ mass law departure from theory seen at $45 \mathrm{kHz}$ would be akin to a $2.8 \mathrm{~dB}$ insertion loss. However, the increase in the transmission for the mass law region at $65 \mathrm{kHz}$ would correspond to a $-5.7 \mathrm{~dB}$ insertion loss departure from theory. Though accurate determination of the mass law transmission levels is not the focus of this thesis, it may warrant further consideration to determine the exact reason for the departure from theory, though again this departure may be due to experimental error. 


\section{Chapter 5}

\section{Conclusions}

An experimental setup has been developed to measure the angular dependence of airborne sound transmission through thin bars. The coincidence effect has been shown to be observable in finite bars with no cavity backing. Coincidence has also been observed using continuous waves, i.e. in steady state. Finally, further experimental evidence has been given to suggest that the theory for transmission near grazing angles of incidence does not match experimental findings with departures on the order of $50 \mathrm{~dB}$.

The use of a cavity to observe coincidence in finite plates described by Bhattacharya et

$a l .{ }^{5}$ is shown to be unnecessary when using an array of microphones. The matching of propagating flexural wave numbers to propagating acoustic wave numbers is possible in finite bars, even when using continuous wave excitation. This wave number matching is the basis for the standard coincidence effect predicted for infinite plates. 
Most of the recent studies about the coincidence effect have used short-duration pulsed waves to measure transmission. . The use of continuous waves increases the SNR over pulsed waves but requires observing coincidence in steady state. The data presented here show evidence of the coincidence effect using continuous waves.

Transmission near grazing angles has also been addressed. Experimental data further verify that theory for infinite plates and experimental results for finite bars diverge at angles near grazing incidence. The implication of this result merits further investigation. An experimental setup designed to measure the transmission at grazing incidence would be useful in determining the causes of the divergence at these angles.

The measurement system described in this thesis has several drawbacks. First, the $2.5^{\circ}$ angular resolution is limiting in its ability to distinguish the coincidence peaks. A setup with better resolution could improve the quality of the results. Second, the setup requires multiple microphones rather than the single transducer used by Davies and Gibbs. ${ }^{7}$ Third, the effect of the foam baffle and duct tape is still unknown, and future work could explore this. Fourth, the aluminum bar was still wide enough to contain several flexural wavelengths, and measuring transmission through narrower bars could lead to improvements in the results.

A potential future use of the measurement system developed for this research is the determination of material properties. The concept of using transmission of sound, especially ultrasonic sound, to determine properties of the material under test has already been treated in the literature, ${ }^{18-20}$ but not specifically by tracking the coincidence angle versus frequency to extract these properties. Using the technique developed for this research, the coincidence angle as a function of frequency can be measured. Since the mass per unit area can easily be measured and 
calculated, the bending stiffness term can be determined by fitting a theoretical curve to this data. Appendix D demonstrates this proof of concept in more detail. 


\section{References}

1. B. E. Anderson, W. J. Hughes and S. A. Hambric, "Grating lobe reduction in transducer arrays through structural filtering of supercritical plates," J. Acoust. Soc. Am. 126 (2), 612-619 (2009).

2. L. Cremer, "Theorie der Schalldämmung dünner Wände bei schrägen Einfall," Akust. Z. 7, 81-104 (1942).

3. G. Lamb, Jr., "Transmission of a Spherical Wave through a Thin Elastic Plate (A)," J. Acoust. Soc. Am. 28 (1), 162 (1956).

4. E. G. Eichler and R. F. Lambert, "Sound Transmission through Thin Plates at Oblique Incidence (A)," J. Acoust. Soc. Am. 29 (11), 1253 (1957).

5. M. C. Bhattacharya, R. W. Guy and M. J. Crocker, "Coincidence effect with sound waves in a finite plate," J. Sound Vib. 18 (2), 157-169 (1971).

6. M. C. Bhattacharya and M. J. Crocker, "Forced vibration of a panel and radiation of sound into a room," Acustica 22, 275-294 (1970).

7. J. C. Davies and B. M. Gibbs, "The oblique incidence measurement of transmission loss by an impulse method," J. Sound Vib. 74 (3), 381-393 (1981).

8. A. C. Raes, "Static and Dynamic Transmission Losses of Partitions," J. Acoust. Soc. Am. 35 (8), 1178-1182 (1963).

9. M. M. Louden, "The single-pulse method for measuring the transmission characteristics of acoustic systems," Acustica 25, 167-172 (1971).

10. B. E. Anderson, W. J. Hughes and S. A. Hambric, "On the steering of sound energy through a supercritical plate by a near-field transducer array," J. Acoust. Soc. Am. 123 (5), 2613-2619 (2008).

11. F. Fahy and P. Gardonio, Sound and Structural Vibration: Radiation, Transmission and Response, 2nd ed. (Elsevier/Academic, 2007), pp. 284-295. 
12. B. H. Sharp, "Prediction methods for the sound transmission of building elements," Noise Cont. Eng. 11 (2), 53-63 (1978).

13. F. G. Leppington, K. H. Heron, E. G. Broadbent and S. M. Mead, "Resonant and NonResonant Acoustic Properties of Elastic Panels. II. The Transmission Problem," Proc. R. Soc. A 412 (1843), 309-337 (1987).

14. M. Villot, C. Guigou and L. Gagliardini, "Predicting the acoustical radiation of finite size multi-layered structures by applying spatial windowing on infinite structures," J. Sound Vib. 245 (3), 433-455 (2001).

15. B. E. Anderson, "Grating lobe reduction in transducer arrays through structural filtering of supercritical plates," Ph.D. thesis, The Pennsylvania State University, University Park, PA, (2006).

16. T. Jenny and B. E. Anderson, "Ultrasonic anechoic chamber qualification: Accounting for atmospheric absorption and transducer directivity," J. Acoust. Soc. Am. 130 (2), EL69-EL75 (2011).

17. V. M. Albers, Underwater Acoustics Handbook - II. (The Pennsylvania State University Press, State College, PA, 1965), pp. 23, 180-205.

18. R. J. Urick, Principles of underwater sound. (McGraw-Hill, 1983), pp. 31-70.

19. D. T. Blackstock, Fundamentals of physical acoustics. (Wiley, 2000), pp. 495-506.

20. L. E. Kinsler, A. R. Frey, A. B. Coppens and J. V. Sanders, Fundamentals of acoustics. (Wiley, 2000), pp. 195-199, 526.

21. B. E. Anderson, B. F. Moser and K. L. Gee, "Loudspeaker line array educational demonstration," J. Acoust. Soc. Am. (Accepted and in press) (2011).

22. Y. A. Balilah and B. M. Gibbs, "The measurement of the transmission loss of single leaf walls and panels by an impulse method," J. Sound Vib. 123 (2), 229-245 (1988).

23. J. Farley and B. E. Anderson, "Ultrasonic airborne insertion loss measurements at normal incidence (L)," J. Acoust. Soc. Am. 128 (6), 3305-3307 (2010).

24. M. Castaings and B. Hosten, "Air-coupled measurement of plane wave, ultrasonic plate transmission for characterising anisotropic, viscoelastic materials," Ultrasonics 38 (1-8), 781-786 (2000).

25. M. Castaings, B. Hosten and T. Kundu, "Inversion of ultrasonic, plane-wave transmission data in composite plates to infer viscoelastic material properties," NDT\&E Int. 33 (6), 377-392 (2000). 
26. T. E. Gomez, B. Gonzalez and F. Montero, "Paper characterization by measurement of thickness and plate resonances using air-coupled ultrasound," presented at the Ultrasonics Symposium, 2002. Proceedings. 2002 IEEE, 2002. 


\section{Appendix A}

\section{LabVIEW Code}

\section{A.1 Tutorial}

The LabVIEW code developed for the measurements presented in this thesis is useful for further research on angular dependent sound transmission and, potentially, for other applications, including directivity measurements of transducers. For this reason, a brief tutorial on using this code will be given in this appendix.

In order for the LabVIEW virtual instrument (VI) to work correctly, the turntable must be connected to the parallel port. A National Instruments (NI) data acquisition (DAQ) controller (the PXI, for example) must also be correctly connected. This can be checked using the NI Measurement and Automation Explorer (MAX). Note that the PXI must be connected and turned on before the controlling computer is turned on. Once this hardware is in place, the VI can be opened. 
A screen shot of the front panel of the LabVIEW virtual instrument (VI) used in making angular dependent transmission measurements is given in Fig. A.1. Several of the input parameters have been labeled and will be discussed. When the run button is first pressed, the VI will check to make sure that the turntable is connected properly. It does this by setting the turntable in motion (turning $\mathrm{ON}$ the turn forward pin) and waiting for the motion pin to alternate ON and OFF. If the turntable is connected correctly, the VI will wait for the turntable to return to $0^{\circ}$, at which time it will stop the motion. The status bar will then read, "The turntable is now reset to zero." If the turntable is not connected properly, the three LEDs under the angle gauge will blink, and the VI will not function. If this happens, stop the VI using the button on the toolbar and reconnect the turntable. Note that a good starting position (before the VI is started) for the turntable is a few degrees before $0^{\circ}$, so that when the VI starts it reaches $0^{\circ}$ quickly and prevents cords from tangling.

Once the turntable is reset to zero, the user may set the input parameters:

a) Sampling Rate: This sets the sampling rate for the DAQ hardware. The user must verify the capacity of the hardware.

b) Block Size: This sets the length of each block recorded.

c) Number of Blocks: This determines the number of blocks recorded at each rotation angle.

d) Buffer Size: This sets the hardware buffer. The value here is dependent on the application, but a typical buffer size is about twice the length of data being recorded.

e) Input source channel: This is the hardware channel to which the source output is connected. This was done in order to compute transfer functions of microphone inputs to 
the output. A microphone recording the source output may be used here to better monitor the source output, but the VI would need to be reconfigured to provide ICP power.

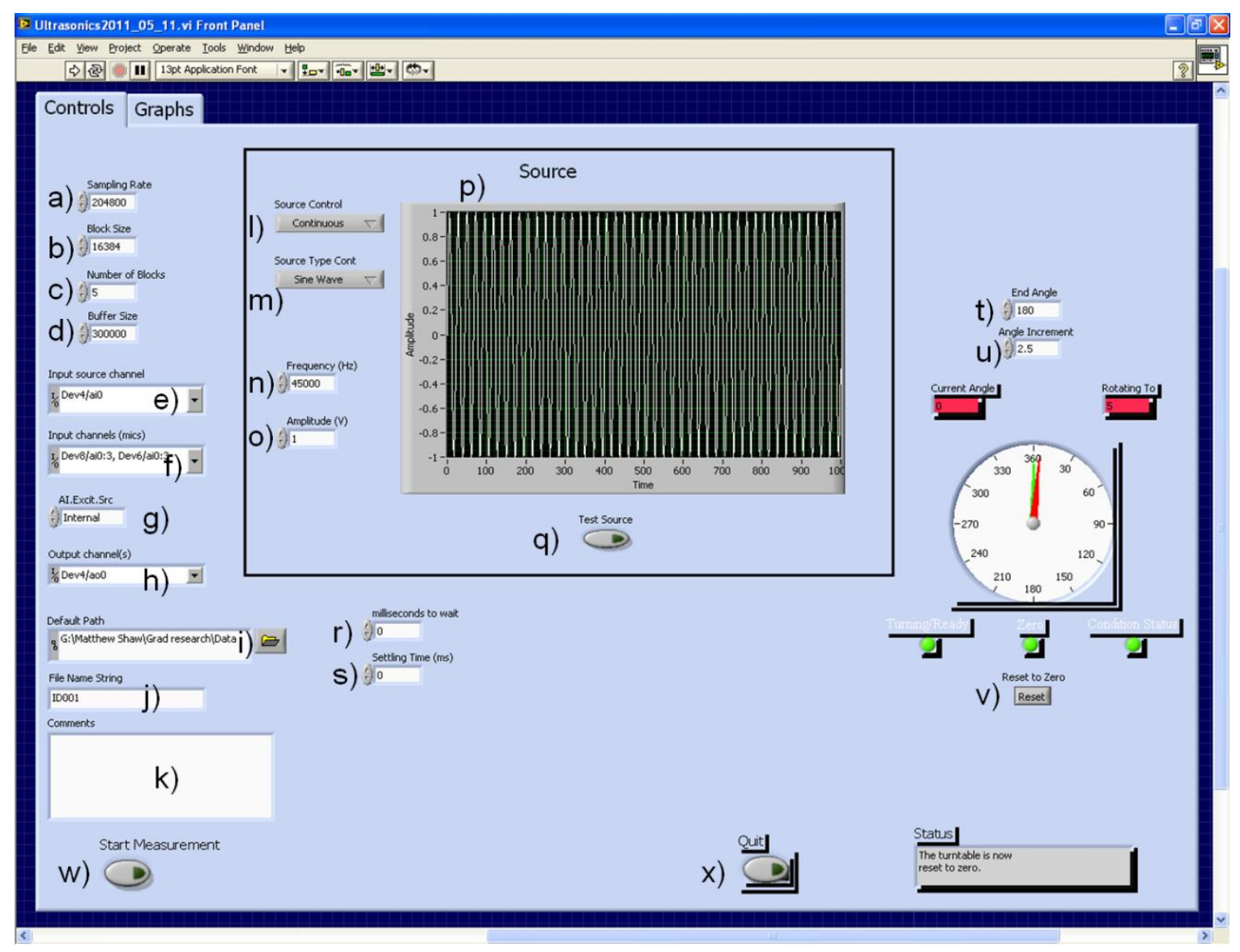

FIG. A.1. Screen shot of LabVIEW VI front panel.

f) Input channels (mics): These are the channels which are connected to microphones. This determines the number of channels recorded.

g) AI.Excit.Src: This is the source of the polarization voltage or current for the microphones. If the microphones require an external power supply, this must be changed to "External." If the PXI must provide ICP power, then use the "Internal" option. 
h) Output channel(s): This is the hardware channel to which the source (speaker, piezoelectric, etc.) is connected. Generally, the output of this channel is branched to the source and to "Input source channel."

i) Default Path: This is the folder where the data will be stored.

j) File Name String: This is the identifier on the beginning of the recorded data files. The VI is currently set up to name the files "File Name String" + "Channel Number" + "Rotation Angle" + "Block Number".

k) Comments: This is where the user can insert comments about the specific measurement being taken. A text file is created for each time the user presses "Start Measurement."

1) Source Control: The user may use either a continuous noise source or a pulsed wave source. Currently, the "Block" wave control is undeveloped.

m) Source Type Cont: This determines whether a single-frequency sine wave or white noise is used as a source. This changes if "Source Control" is changed to "Block."

n) Frequency $(\mathrm{Hz})$ : This sets the frequency of the sine wave sent to the speaker. This is removed if "White Noise" is selected for "Source Type Cont."

o) Amplitude (V): This sets the amplitude of the sine wave sent to the speaker. The user must be careful to not send too much voltage to the source transducer.

p) Waveform graph: This displays the type of signal being sent to the speaker.

q) Test Source: This button may be pressed to send a 1 s sample of the source signal to the speaker.

r) Milliseconds to wait: This must remain at zero. This parameter was used in troubleshooting and is no longer necessary. 
s) Settling Time (ms): This sets the settling time between rotation angles. This was used to allow the bar and foam setup on the turntable to settle after rotating the turntable.

t) End Angle: This determines how far from $0^{\circ}$ the turntable takes measurements. For the purposes of transmission, $180^{\circ}$ was sufficient. For a full directivity measurement, $360^{\circ}$ would be more appropriate.

u) Angle Increment: This determines the angular resolution of the measurement. The options range from $2.5^{\circ}$ to $90^{\circ}$. The turntable rotates in the direction of increasing angle (clockwise) by this increment until it reaches the value specified in "End Angle."

v) Reset to Zero: This button may be pressed after completing a measurement to return the turntable to $0^{\circ}$. It sends the turntable in a counter clockwise (backwards) rotation.

w) Start Measurement: This button is pressed to begin a measurement. This will be described further.

x) Quit: This button may be pressed to quit execution of the VI. Caution must be taken when pressing this button, as its functionality is not completely tested. If the Quit button is clicked during a measurement, then it may take a few moments to actually stop. The turntable will then need to be manually rotated clockwise back to $0^{\circ}$, or past $0^{\circ}$.

Once the VI is running, the user has set all of the inputs, and the turntable has been reset to zero, the user may push the "Start Measurement button," at which time the VI will show the "Graphs" tab of the front panel. On the right side are the angle gauge, "Current Angle" and "Rotating To" indicators, the LED indicators, and the status bar as seen in Fig. A.1. These allow the user to monitor the rotation progress of the measurement. On the left side are two plots, the top one for displaying the time waveforms of each block as it is recorded, and the bottom one for 
displaying the spectra of the corresponding waveforms displayed above. A binary file is created and written every time an block is recorded.

Once the measurement is complete, the VI will return to the "Controls" tab of the front panel, at which time the user may press the "Reset to Zero" button to rotate the turntable counter clockwise back to $0^{\circ}$. At this time, the measurement is complete, and binary files will have been created. These files can be read into MATLAB using a program script similar to the one in Appendix B. To start a new measurement, the user must modify the "File Name String" and any input parameters that are different for the next measurement and press "Start Measurement" again.

\section{A.2 Block Diagram}

This section will give a brief overview of the various parts of the block diagram. A view of the full block diagram is given in Fig. A.2, with boxes indicating sections that will be zoomed in and discussed in greater detail.

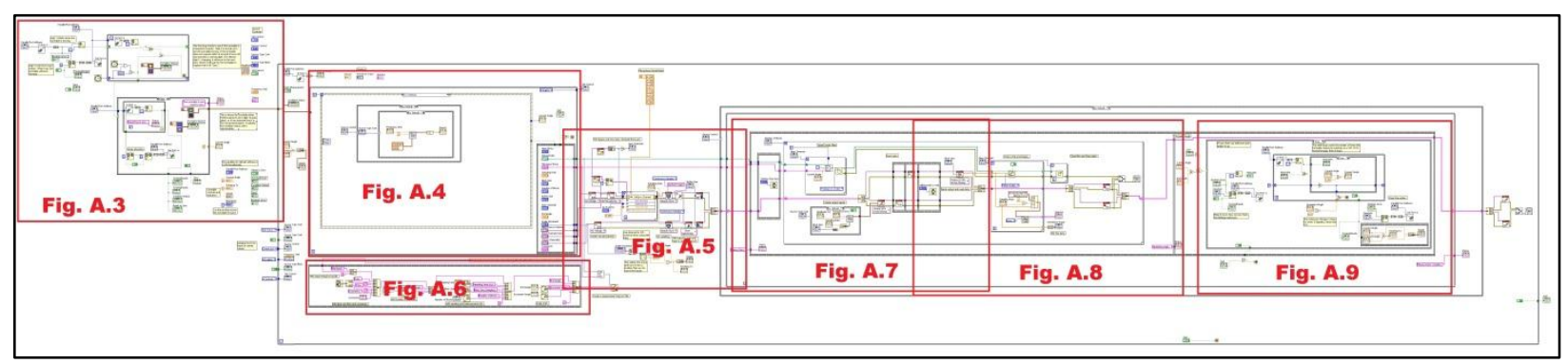

FIG. A.2. Screen shot of the entire LabVIEW VI block diagram. 


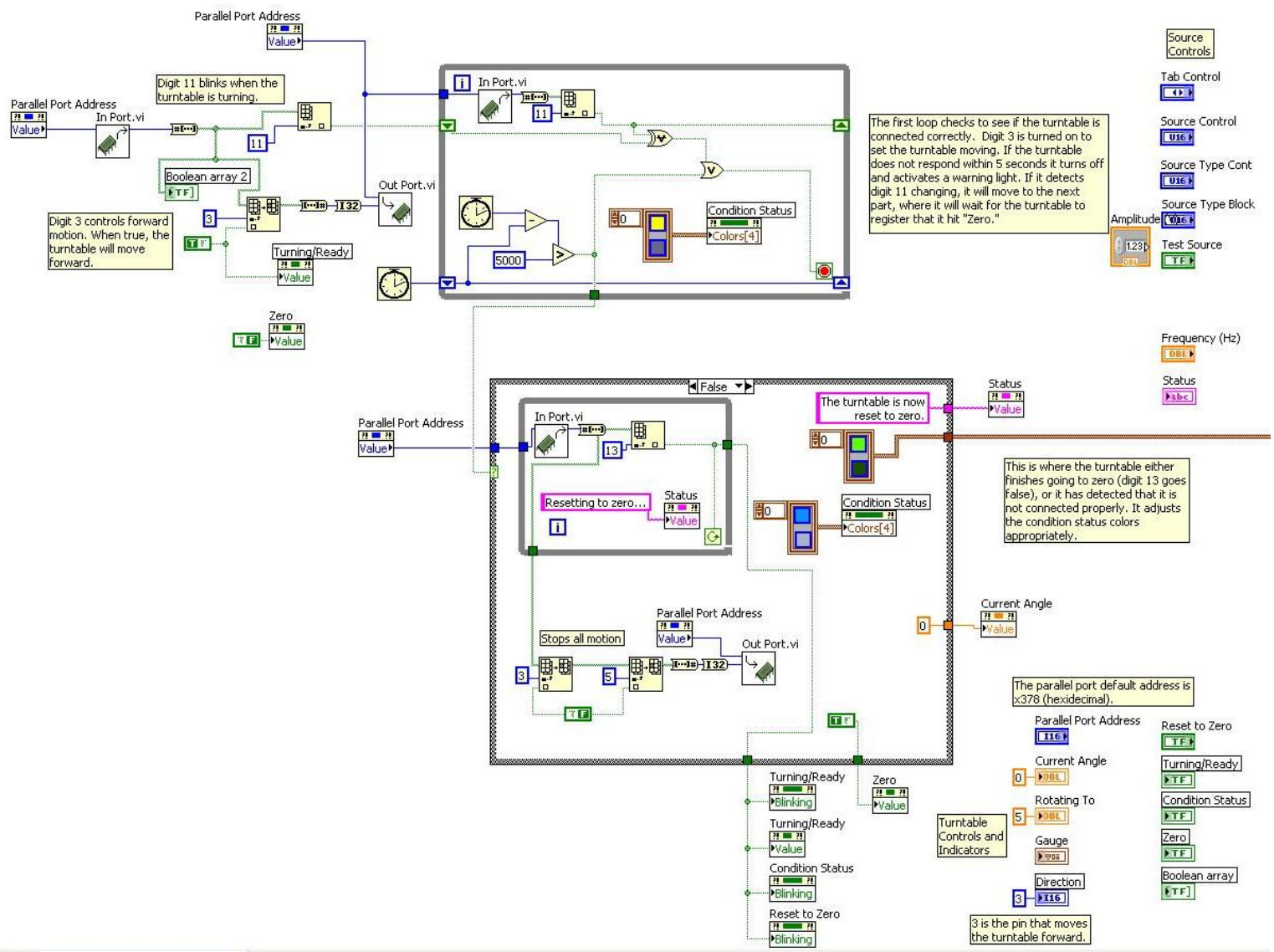

FIG. A.3. The turntable initialization section of the VI block diagram.

The first part of the VI is shown in Fig. A.3. This part checks that the turntable is correctly connected to the computer. The top left section sets the turntable in motion. The top while loop observes the pin on the parallel port that alternates on and off when the turntable is in motion. If this occurs, data flow exits that loop and the second loop stops the motion when the turntable returns to $0^{\circ}$. At this point, the VI moves into the main while loop execution. 


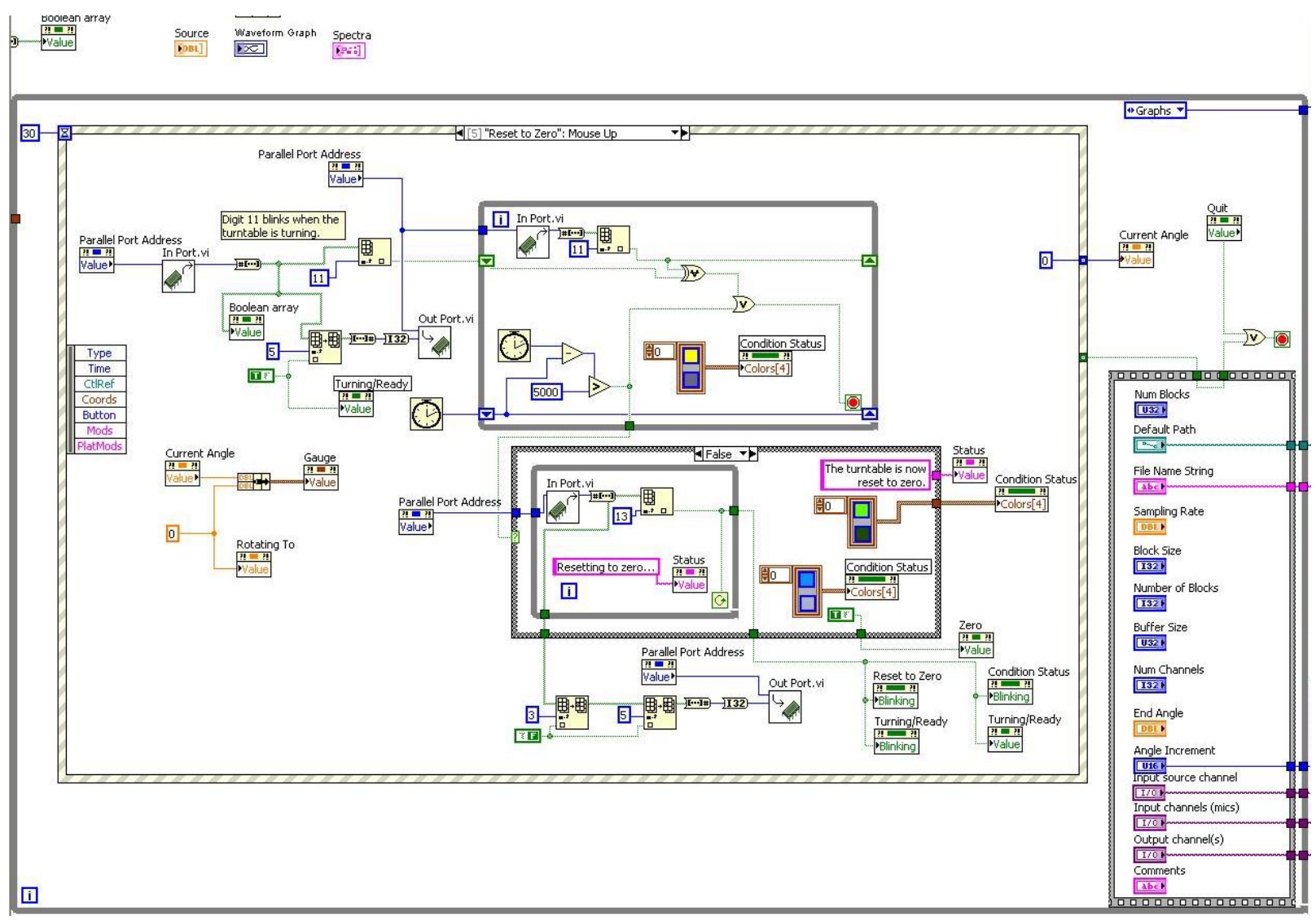

FIG. A.4. The while loop in the VI block diagram that waits for the user to begin the measurement.

When the VI moves into the main program loop, the first sub loop contains an event structure (composed of stacked frames each executed at different times when the corresponding button is pressed) that waits for the user to press a button. This sub loop is shown in Fig. A.4. On timeout (if the user presses nothing), the event structure plots the source signal in the waveform graph on the front panel. Shown in the figure is the case when the user presses the "Reset to Zero" button, which is similar to the initialization section shown in Fig. A.3. When the user presses "Start Measurement," the VI initializes the variables on the right side and exits into the DAQ initialization stage. 


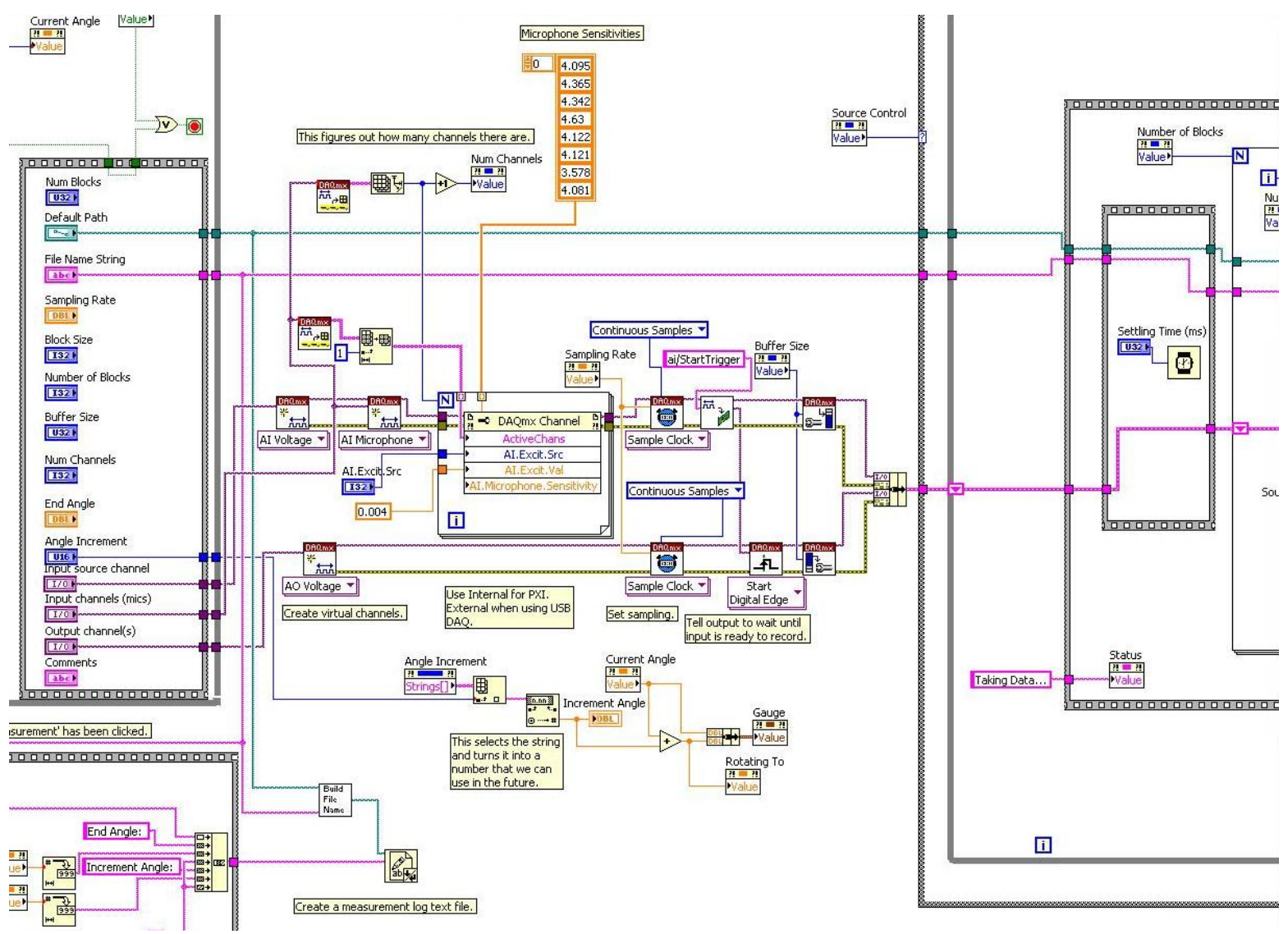

FIG. A.5. DAQ initialization in the VI block diagram.

Figure A.5 shows the DAQ initialization. The analog input and analog output virtual channels are created in the middle section. The microphone sensitivities, given in units of $\mathrm{mV} / \mathrm{Pa}$, are inserted in the orange-colored array in Fig. A.5, meaning that recorded data are in pressures. The sampling frequency and buffer are initialized, and the chosen increment angle is converted to a usable number (instead of a string). The very bottom section is where a measurement log text file is created. Figure A.6 shows more about the creation of this log file. 


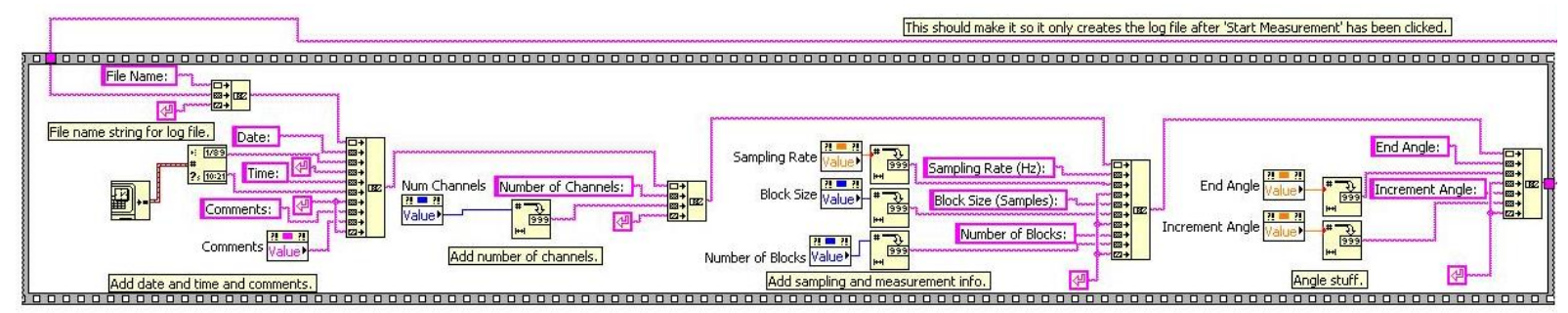

FIG. A.6. Measurement log file compilation in the VI block diagram.

Figure A.6 shows the diagram that sets up the measurement log text file. Every time the "Start Measurement" button is pressed, a log file is created. The various important variables for the measurement are imported into this portion, and each of them is converted to a string. The entire set is concatenated into one long string, which is then written to a text file with the name of the "File Name String" variable. Once the log file has been created and the DAQ tasks are initialized, execution moves into the measurement loop of the VI. 


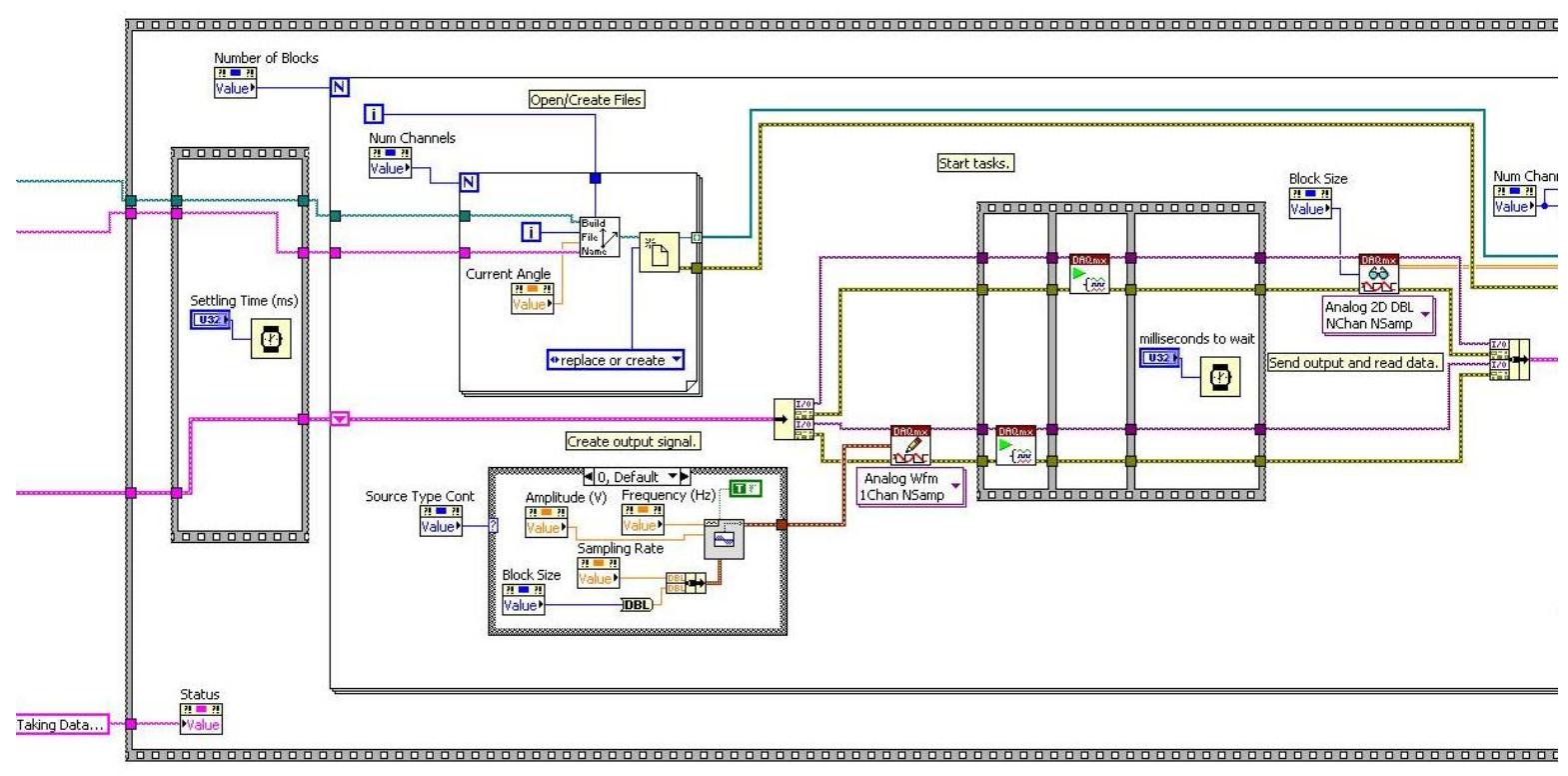

FIG. A.7. The first section of the measurement loop of the VI block diagram.

Figure A.7 shows the first part of the loop where the actual measurement takes place. The data flow first passes through a settling time section. This is important when using a larger plate, as time is required to dampen out the vibrations caused by rotating the turntable. The for loop then executes once for each block being recorded. During the first step in the for loop the VI first creates files for each channel. The output signal is created below that, and the DAQ tasks are started. 


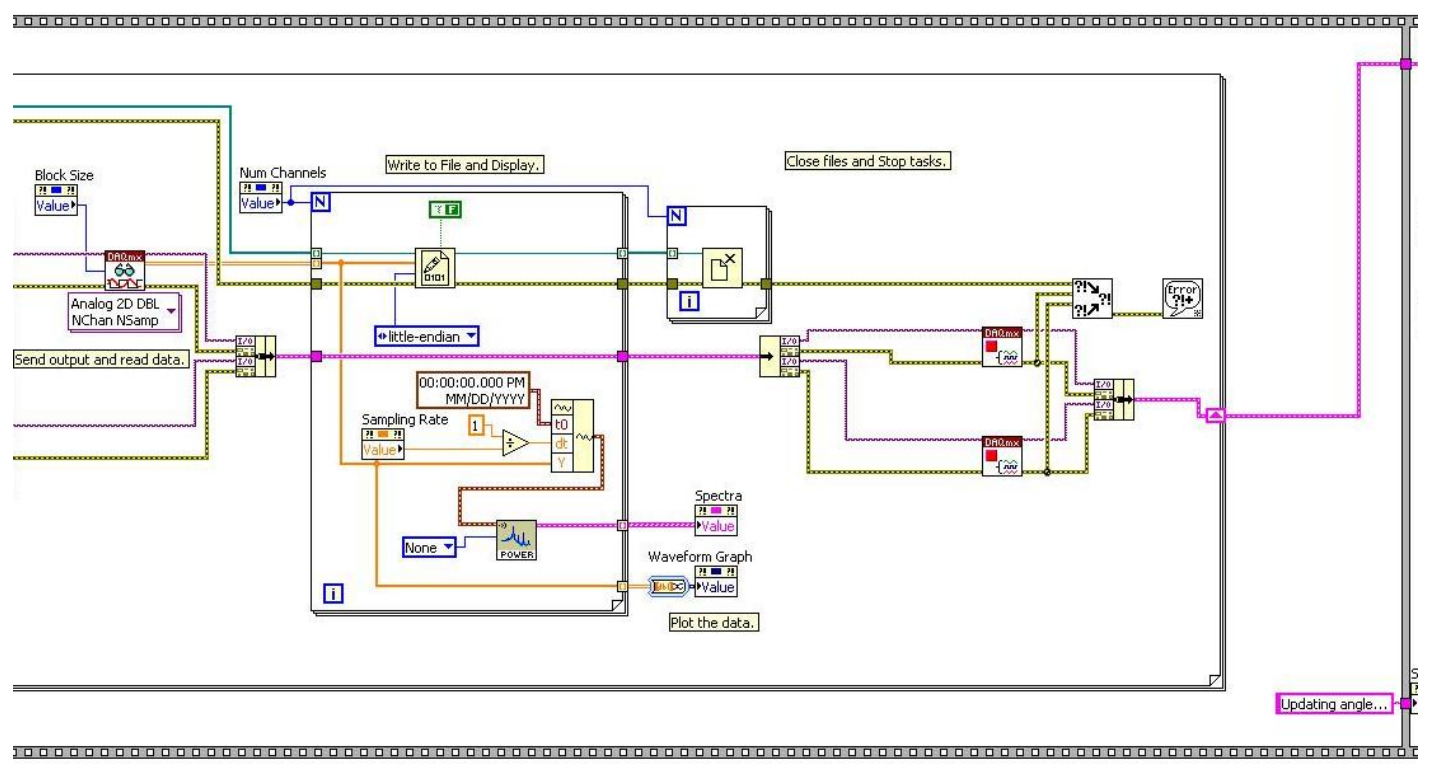

FIG. A.8. The second part of the measurement loop in the VI block diagram.

The next section, shown in Fig. A.8, shows when the data are actually recorded, displayed, and written to file. The far left shows the DAQ acquire command. The data are read in and written to file in the smaller for loop, while the spectra are computed. The files are then closed, the displays are updated with the newly recorded block, and the tasks are stopped. The for loop is then re-executed for each block. 


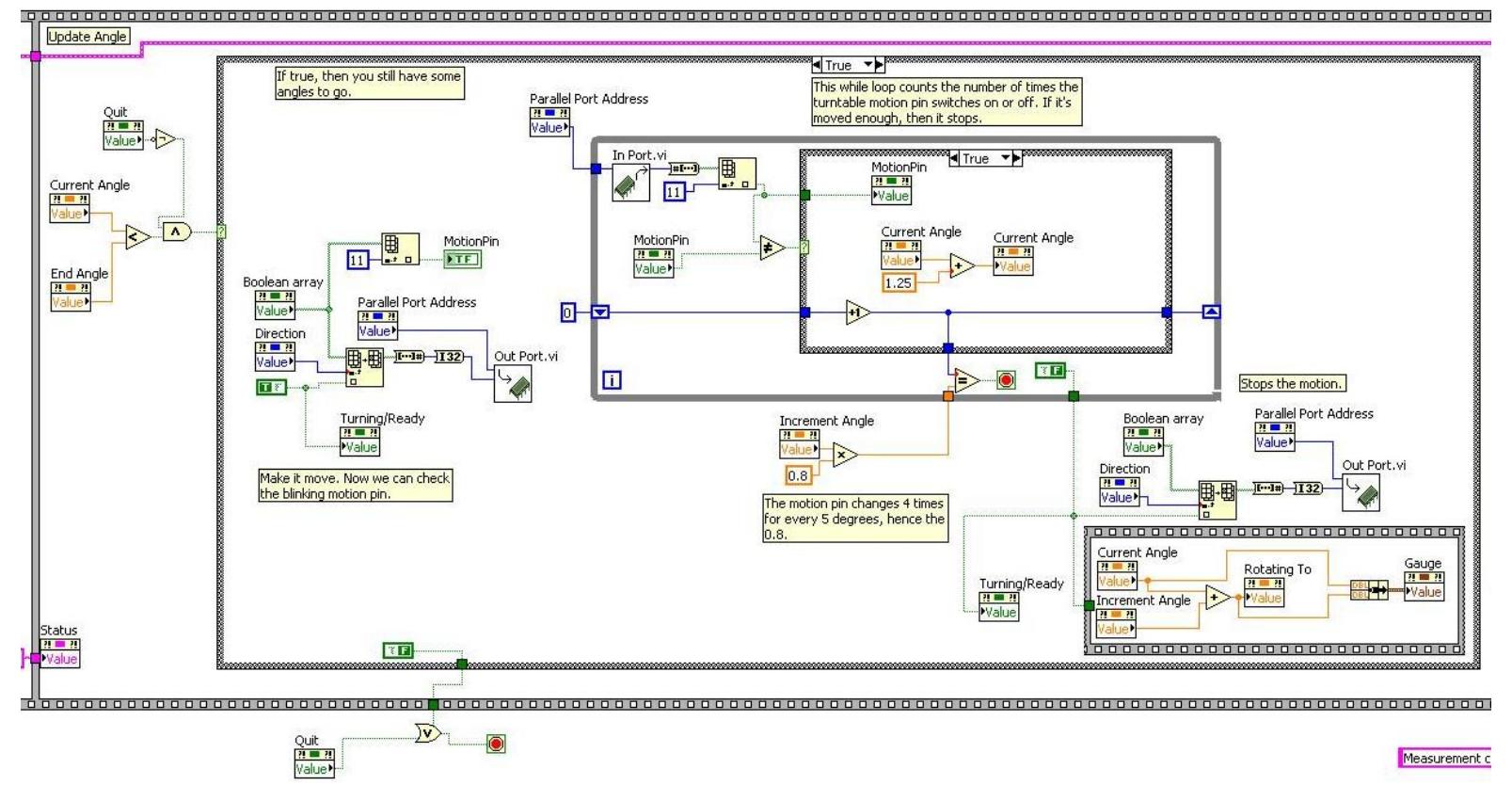

FIG. A.9. The final section of the measurement loop in the VI block diagram.

Once all of the blocks have been recorded and written to file at the current rotation angle, execution moves into the final section of the measurement loop. This section, shown in Fig. A.9, is for updating the angle on the turntable. The VI first determines whether the measurement has reached a conclusion based on the "End Angle" parameter set by the user. If the measurement requires more data at further angles, the turntable is rotated by the amount designated in "Angle Increment." The VI then returns to the section shown in Fig. A.7, where new files are created at the next rotation angle. If the measurement is complete, the loop is exited and execution returns to the loop shown in Fig. A.4. 


\section{Appendix B}

\section{MATLAB Code}

This section will give a brief overview of the various MATLAB files that were used in processing and presenting the data for this research. Each file will be given in its own section.

\section{B.1 Ultrasonicsv2.m}

The purpose of this file is to read in the data recorded by LabVIEW and process it according to the method described in Chapter 3. The first part reads in the data and extracts the magnitude and phase. The second part sums the magnitudes and phases according to standard array beam forming given in Eqs. (3.2) and (3.3). 


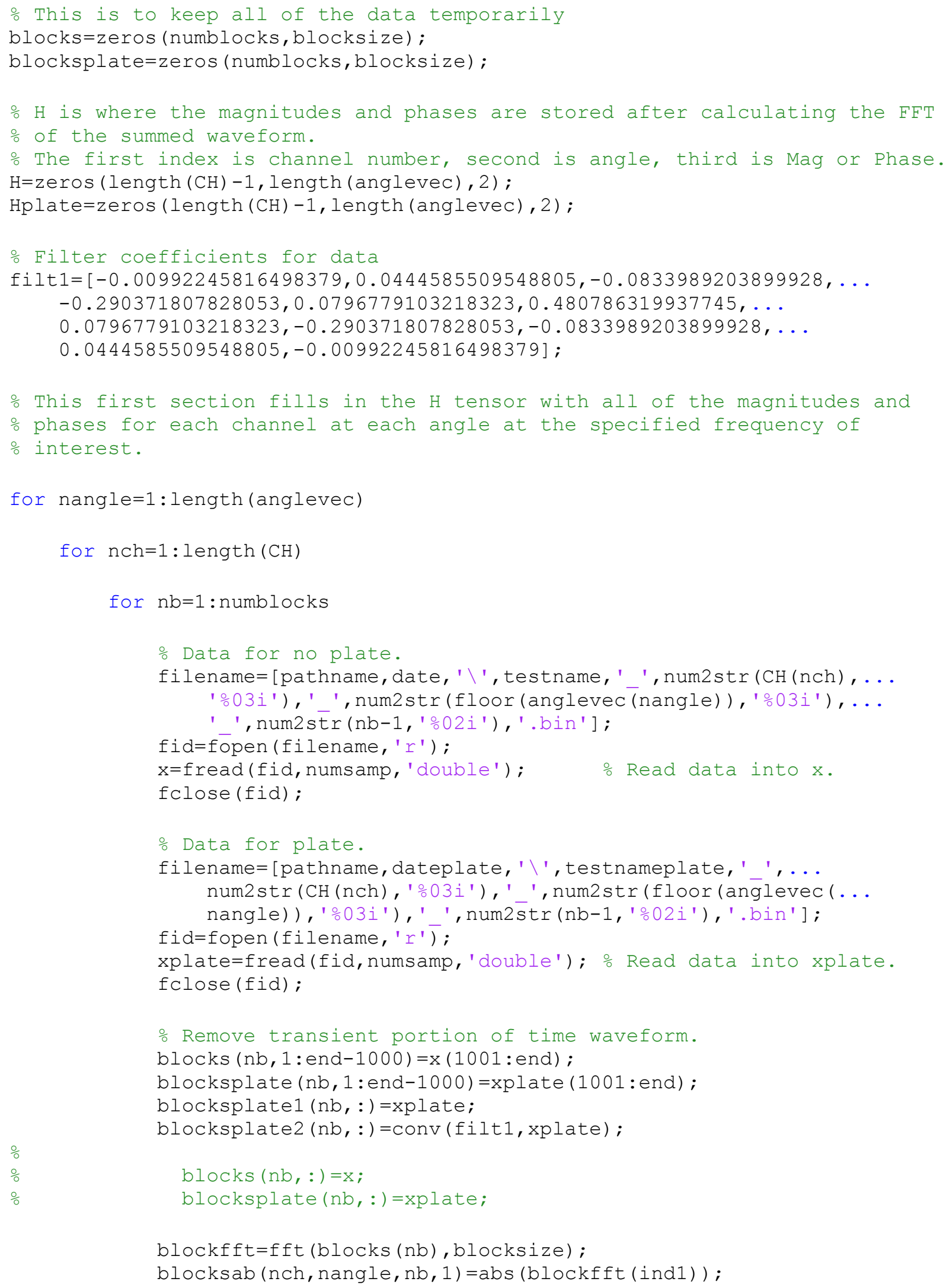


blocksab (nch, nangle, nb, 2) =angle (blockfft (ind1)) ;

blockfftplate=fft (blocksplate (nb), blocksize) ;

blocksabplate (nch, nangle, nb, 1) =abs (blockfftplate (ind1)) ;

blocksabplate (nch, nangle, nb, 2) =angle (blockfftplate (ind1)) ;

end

if nangle $==17$

if $\mathrm{nch}==2$

figure

plot (t,blocksplate2 (1,1:blocksize), 'k')

hold on

plot ( $t$,blocksplate2 (2,1:blocksize), 'b' )

plot ( $t$, blocksplate2 (3,1:blocksize), 'r' )

plot ( $t$,blocksplate2 (4, 1:blocksize) , ' $g^{\prime}$ )

plot ( $t$,blocksplate2 (5, 1 :blocksize), 'm')

hold off

xlabel ('Time (s) ') ; ylabel ('Pressure (Pa) ');

grid on

pause

end

end

if $\mathrm{CH}(\mathrm{nch})==1$

$\mathrm{blocks}=\mathrm{blocks} * 2$;

blocksplate $=$ blocksplate ${ }^{*}$;

end

\% Time-align the blocks to the first block using cross correlation.

for $i \mathrm{i}=1$ : numblocks-1

maxlags $=2{ }^{*} \operatorname{ceil}(\mathrm{fs} / \mathrm{fofint}) ; \quad$ ax possible time lag

$\operatorname{corrtemp}=x \operatorname{corr}($ blocks $(1,:), \operatorname{blocks}(i i+1,:), \operatorname{maxlags})$;

corrtempp $=$ xcorr (blocksplate $(1,:)$, blocksplate $(i i+1,:), \ldots$ maxlags) ;

[valtemp, indtemp] =max ( corrtemp);

[val tempp, indtempp] =max ( corrtempp) ;

shiftt=indtemp-maxlags -1 ;

shifttp=indtempp-maxlags-1;

if shiftt>0

blocks (iit1, : ) = $\operatorname{zeros}(1, \operatorname{shift})$ blocks (iit1, ..

$1:$ blocksize-shiftt) ] ;

elseif shiftt<0

blocks (iit1, : ) = blocks (iit, abs (shiftt) +1:blocksize)... zeros (1, abs (shiftt)) ];

end $\quad$ If shiftt $==0$, then don't make a change.

if shifttp $>0$

blocksplate $(i i+1,:)=[\operatorname{zeros}(1$, shifttp) blocksplate (... iit1, 1 :blocksize-shifttp) ] ;

elseif shiftt $p<0$ 


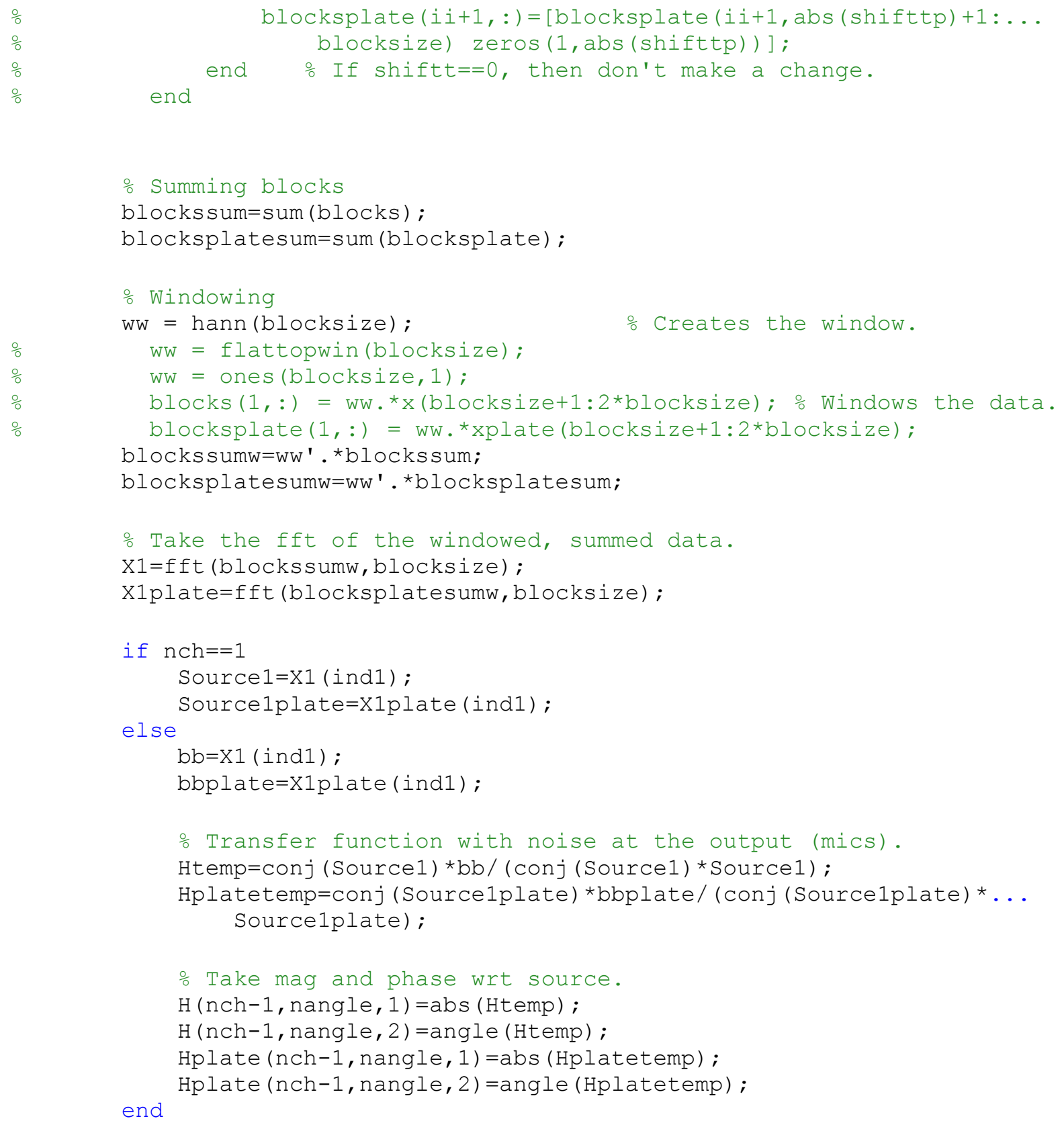




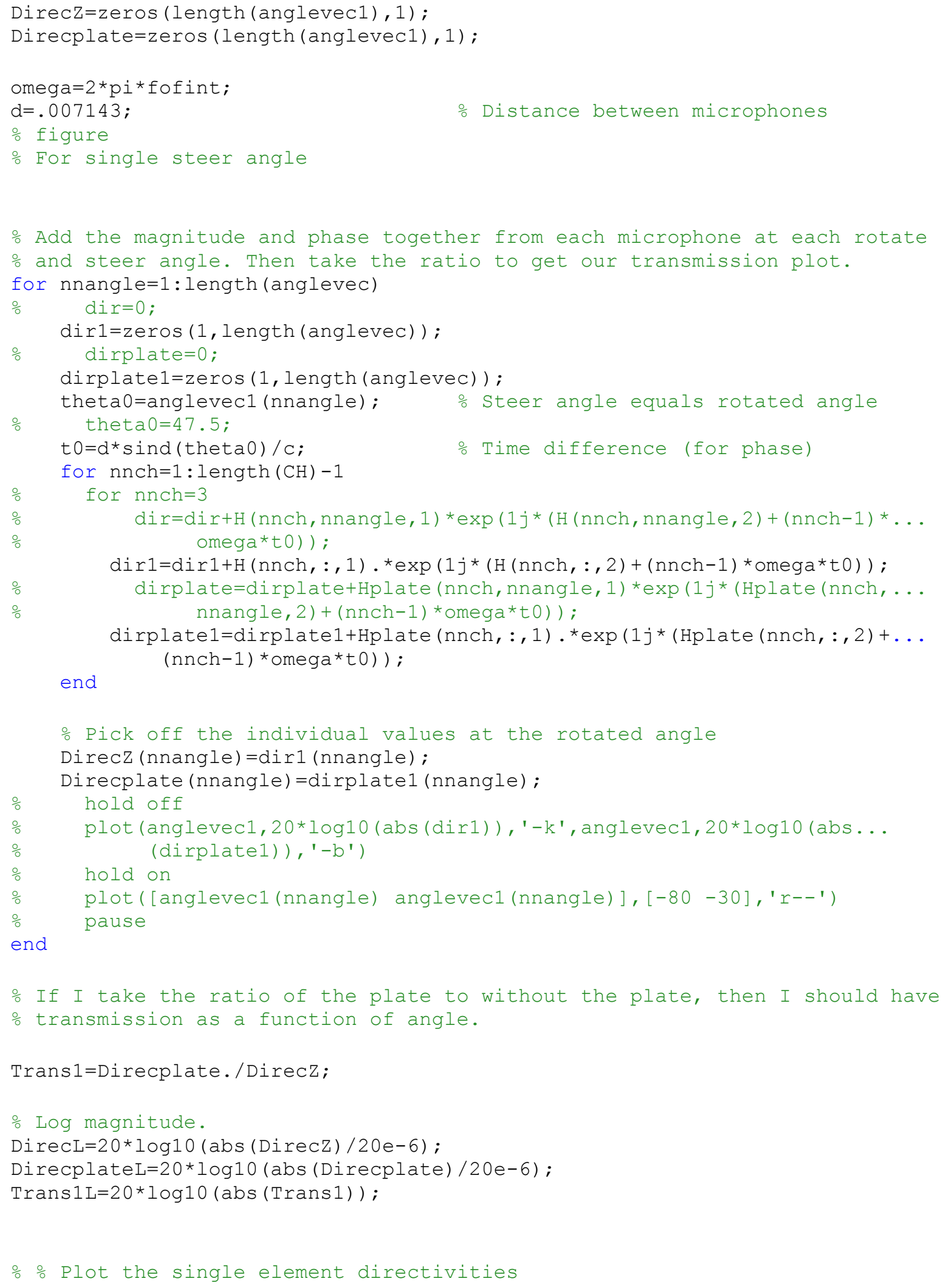




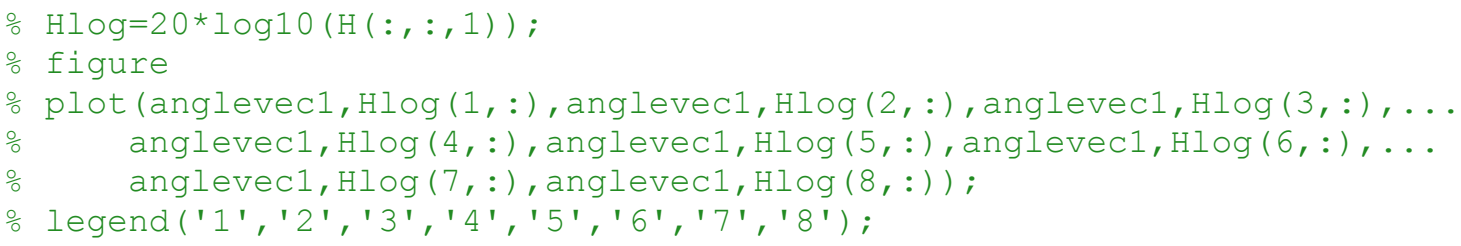

\section{B.2 Trans.m}

This file provides the theoretical transmission curves that are used to compare to experimental results. It also displays the normal incidence mass law for comparison. The equations used in this program are given in Chapter 2. 


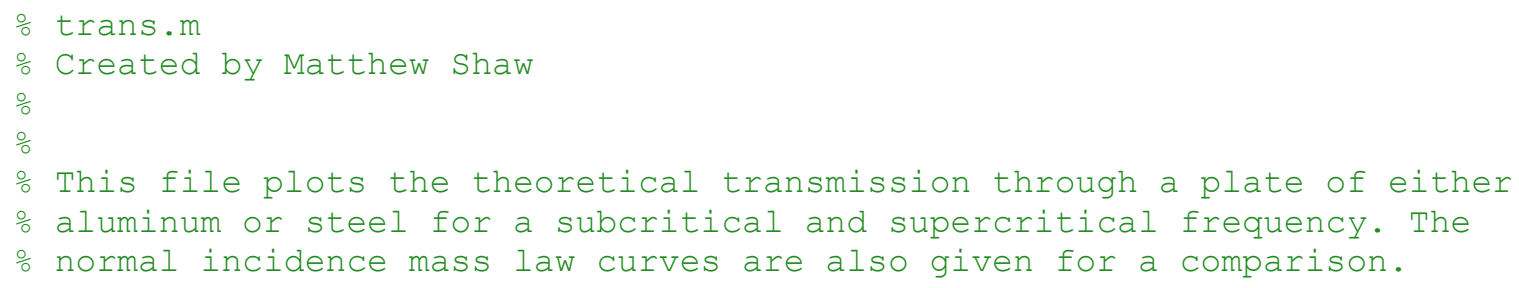




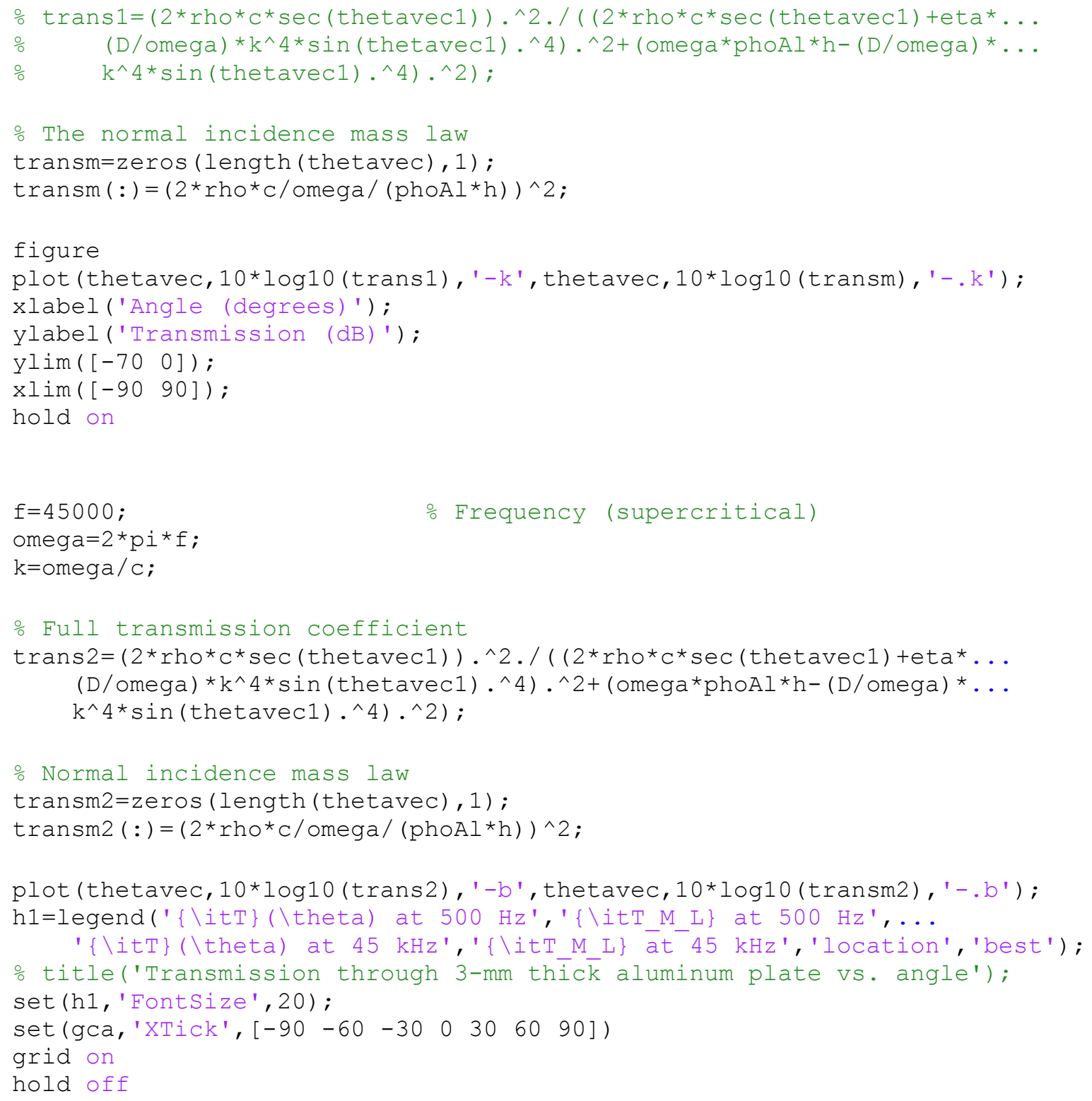

\section{B.3 ThetaCovFreq.m}

This file displays the comparison of measured coincidence frequency to the theoretical curve. This data is shown in Appendix D. 


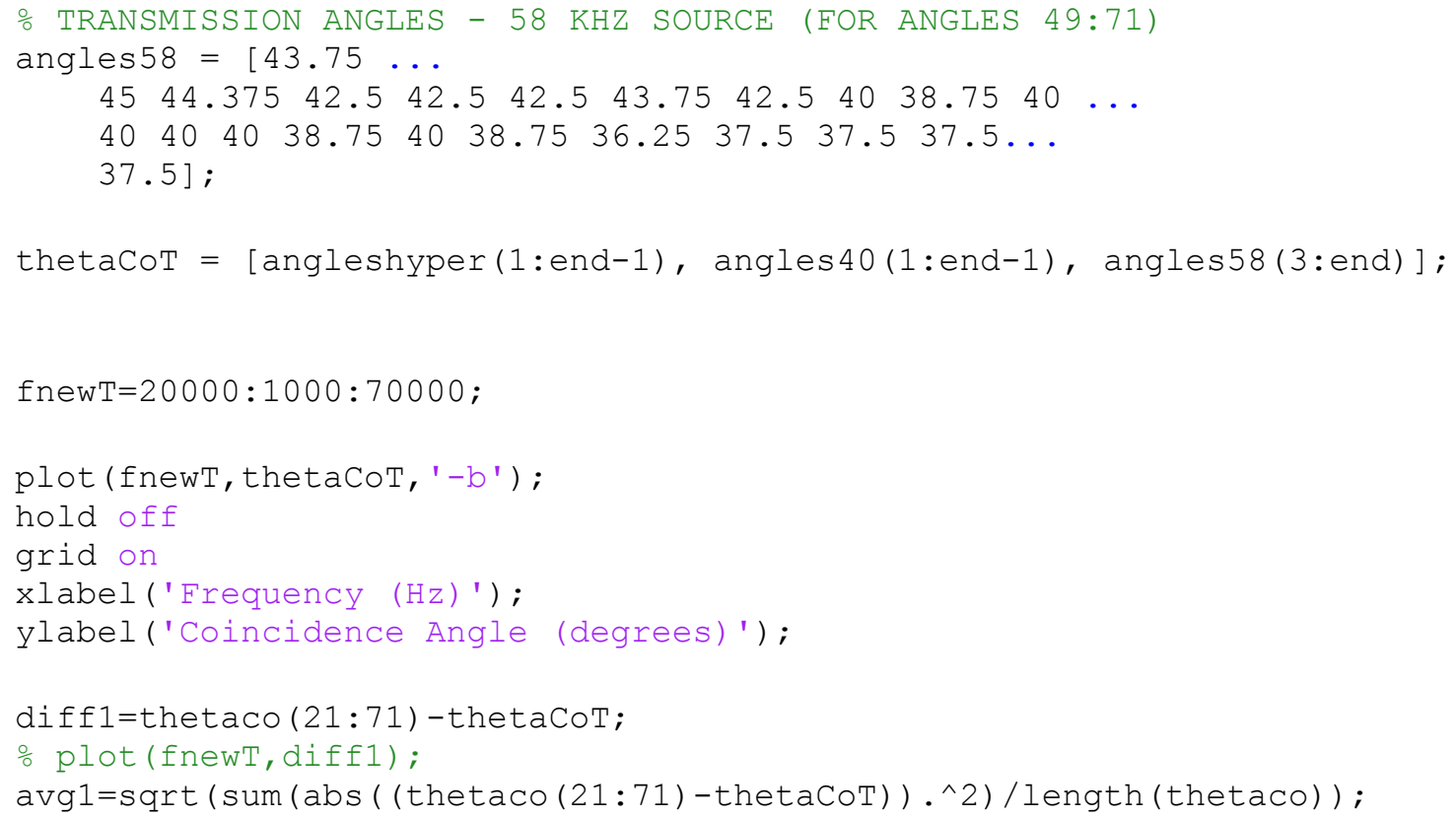




\section{Appendix C}

\section{Experimental History}

Many iterations of the experimental setup were used in order to better understand and improve the quality of the measurement system and determine accurate results. This section is intended to discuss a few of those iterations and the reasoning behind the changes introduced in the setup.

The first plate that was measured with this system was $28 \mathrm{~cm}$ by $40 \mathrm{~cm}$ (11 in by $16 \mathrm{in})$, with a thickness of $0.5 \mathrm{~mm}$. The initial tests were conducted with the plate alone, with no baffle and therefore no attenuation of flanking transmission (due to diffraction). Because it was desired to explore the transmission at grazing incidence, it became important to reduce the diffraction around the sides of the plate. Several different configurations of acoustical treatments were employed to reduce this diffraction by attempting to block direct acoustic paths to the microphones from the side, without these treatments touching the plate. The best results were 
obtained using foam above, below, and to the sides of the microphone array. Figure C.1 shows the data from this measurement.

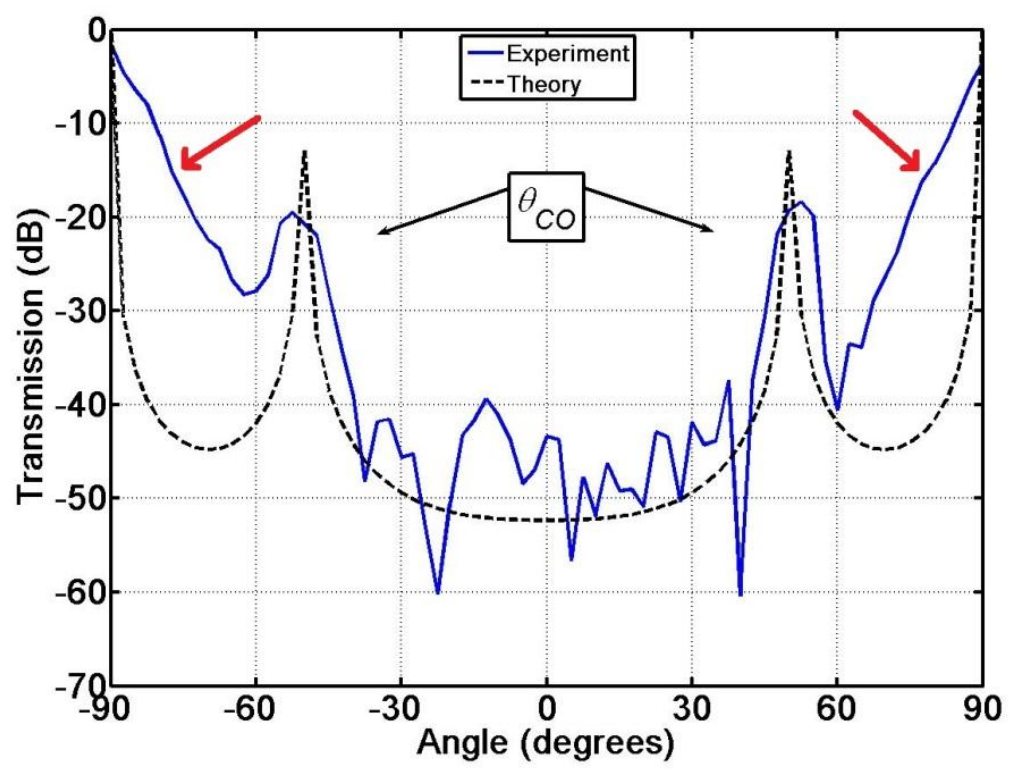

FIG. C.1. Results of transmission during early experiments at $40.5 \mathrm{kHz}$ using foam that was not in direct contact with the plate.

The data in Fig. C.1 were collected using a Mackie HR824 speaker operated at $40.5 \mathrm{kHz}$. The coincidence peaks are visible and reduced in amplitude, similar to the results discussed in Chapter 4. However, as the red arrows identify, the transmission appears to increase as the grazing angles are approached with a fairly linear slope (on a logarithmic scale). This is likely due to diffraction of sound around the sides of the plate and nearly direct sound propagation at the grazing angles. The idea of using a foam baffle, in direct contact with the plate, to reduce the diffracted sound was incorporated into the measurement setup, and new data were collected. The results showed decreased transmission at grazing angles, as expected. The results in Chapter 4 include the foam baffle in direct contact with a bar. 
After the diffraction was reduced, the data still showed significant fluctuations (or seemingly random deviations from the theory) as identified by the red arrows in Fig. 4.3, but they were more frequent and were greater in amplitude, especially in the mass law region, in the measurements with this plate, as shown in Fig. C.1. We postulated that, with a plate, there may be traveling waves propagating in directions other than along the array of microphones which may provide interference with the one-dimensional traveling waves we desired to measure. At this point, it was desired to scan the plate using a Polytec scanning laser Doppler vibrometer in order to observe the wave structure excited in the plate. A $45-\mathrm{kHz}$ sine wave was emitted from the piezoelectric source, and the source was pointed at the plate so that its principle axis made an angle of $45^{\circ}$ to the normal to the plate in the horizontal direction (parallel to the line array). A 17-cm by $8-\mathrm{cm}$ section of the plate was scanned. Figure C.2 shows snapshots of the movie file created during the scans at $0^{\circ}$ phase, $70^{\circ}$ phase, and $140^{\circ}$ phase. Figures C.2(a)-(c) show evidence of a traveling wave in the horizontal direction as evidenced by the vertical ridges (wave fronts) in these plots, but they also show evidence of a vertically traveling wave as evidenced by the faintly visible horizontal ridges (wave fronts). Though not shown here, this same effect was observed when the angle of incidence was from a vertical direction. Figure C.3 shows a scan of the entire plate (with a lower density of points), and the evidence of both vertically and horizontally traveling waves is more clearly seen. 


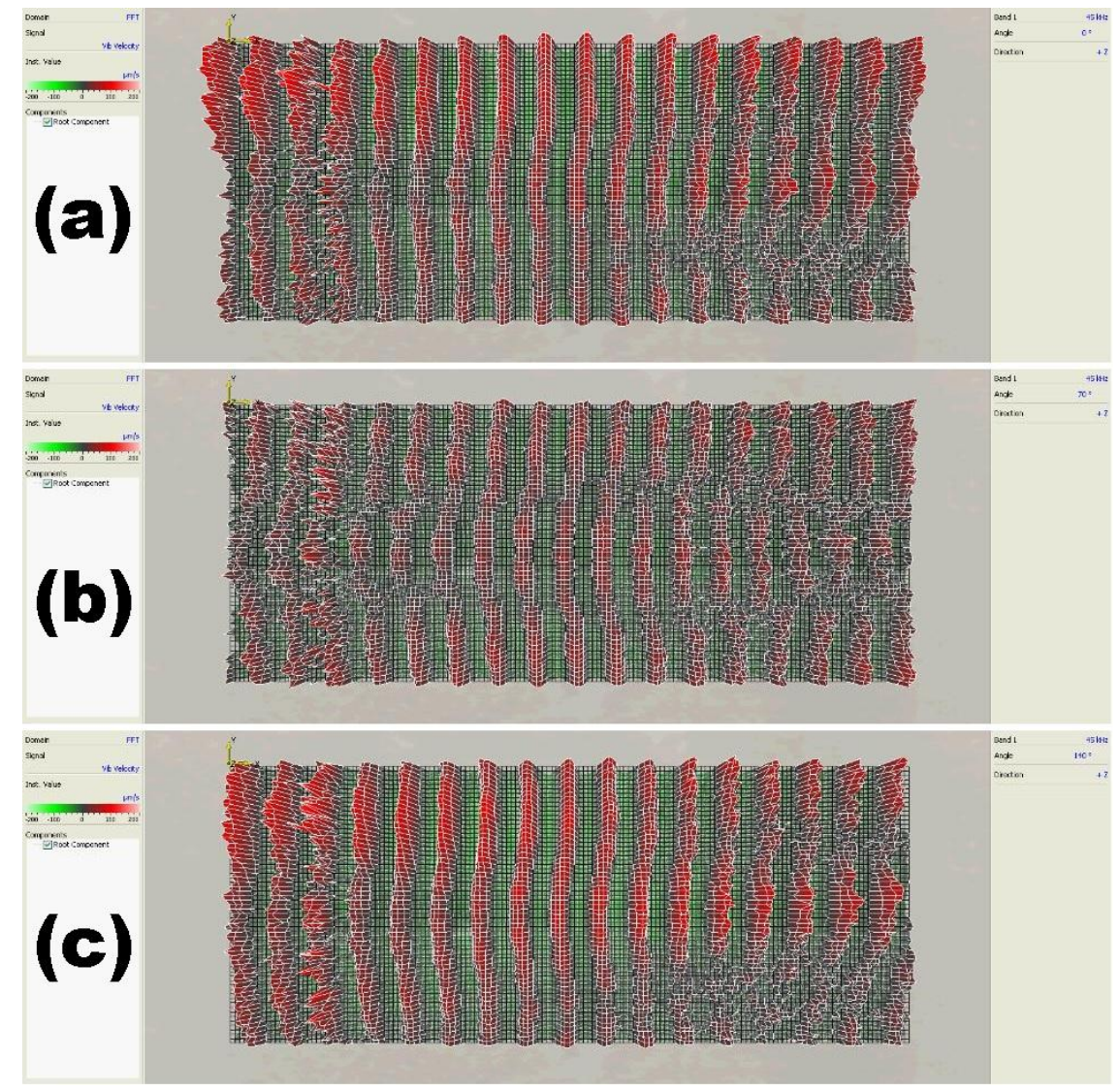

FIG. C.2. Scanning laser vibrometer measurements of a small section of the large plate. (a) $0^{\circ}$ phase (b) $70^{\circ}$ phase (c) $140^{\circ}$ phase. 


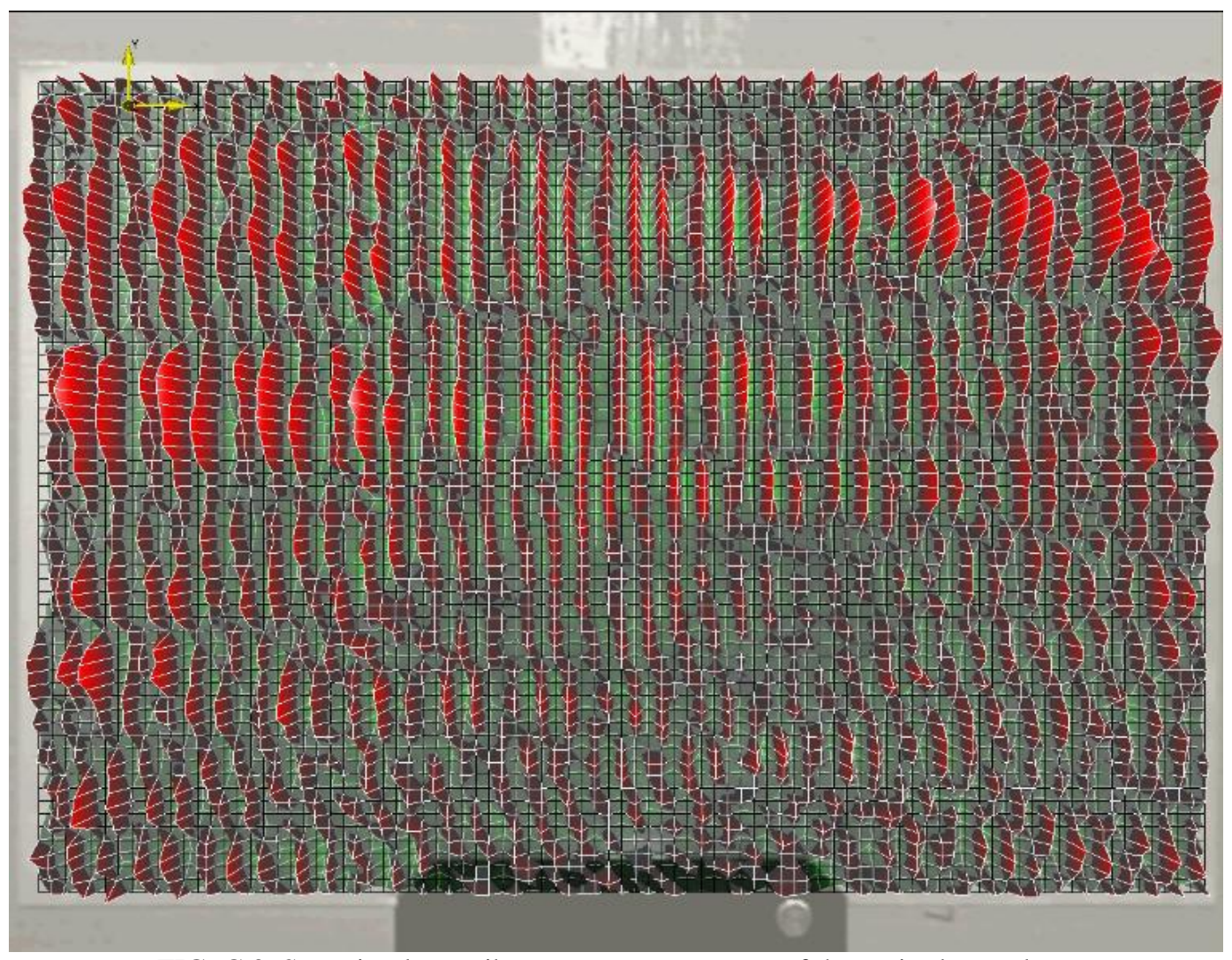

FIG. C.3. Scanning laser vibrometer measurement of the entire large plate.

Because the array was only able to beam form in the horizontal direction, it was believed that the vertically traveling waves are the reason for the large fluctuations in the transmission data. To reduce the effect of the vertically traveling waves, a bar was substituted in place of the plate, with a vertical dimension of $3.8 \mathrm{~cm}$. The bending wavelength at $45 \mathrm{kHz}$ is $1.0 \mathrm{~cm}$. By switching to an aspect ratio closer to that of a bar, the waves were potentially reduced to one dimension. However, the width of the bar is still 3.8 wavelengths in size, so there still may be waves traveling in the vertical direction. Further work could utilize a bar with a smaller width to see if these fluctuations decrease further. 
The use of a foam baffle for the no-bar measurements was explored further to determine its effect on the transmission curve. The question is whether or not the foam baffle should be present for the measurements without the bar, making the presence of the bar the only difference between the two cases. Measurements without the bar were taken both with and without the foam baffle installed, and both were processed according to the sequence described previously (each with the same with-bar data). Figure C.4 shows the comparison of the two cases. The main difference is seen near grazing angles where the transmission is greater with the foam in place for the no-bar measurement. For the results given in Chapter 4, the case without the foam baffle was used because it was believed that the foam baffle did not allow for an unobstructed direct path to the microphones for the no-bar measurements. The incident acoustic wave at grazing incidence drags along $20 \mathrm{~cm}$ of foam before reaching the hole cut out for the microphones and therefore should be attenuated some before it reaches the microphone array. However, even in the case of the foam baffle being present for the no-bar measurement, there may be some attenuation of the incident sound prior to its excitation of the bar, though the wave would only have to travel $6.2 \mathrm{~cm}$ before reaching the taped end of the bar. 


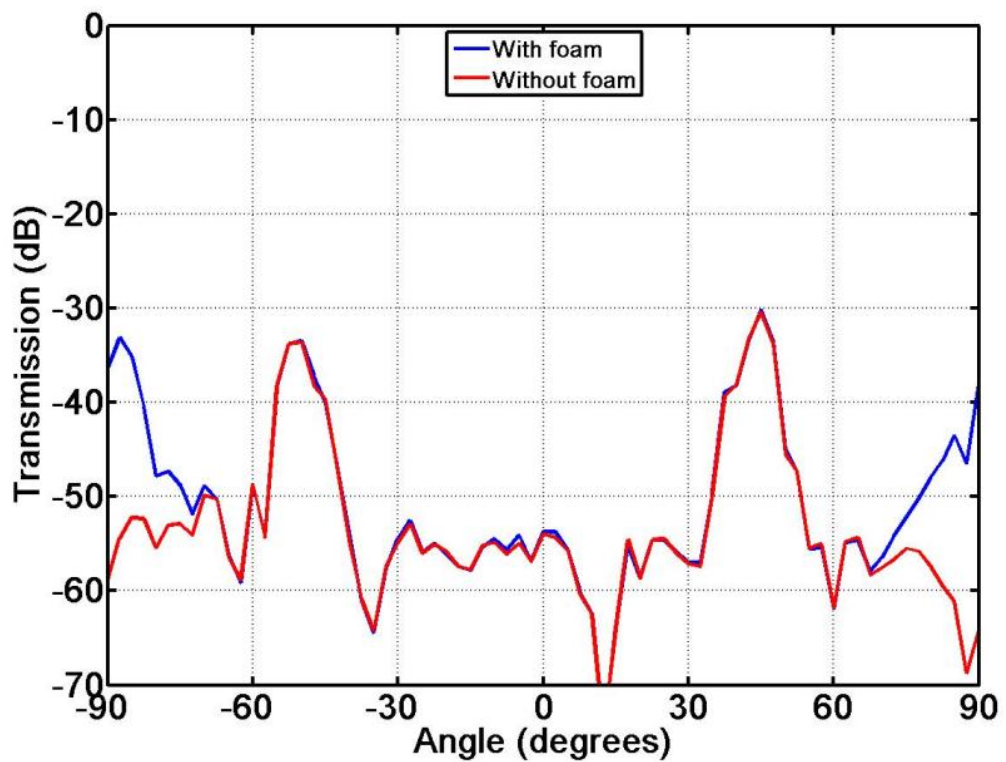

FIG. C.4. Transmission through the $0.5 \mathrm{~mm}$ aluminum bar from experimental data at $45 \mathrm{kHz}$. The no-bar measurements are different for each case in that the foam baffle was present in one and not the other. 


\section{Appendix D}

\section{Material Property Extraction}

The concept of extracting material properties using non-destructive testing (NDT) is not new. While common methods of determining material properties, like elastic moduli and Poisson's ratio, require destruction of the specimen under test, several studies have used

ultrasonic acoustic excitation and transmission to measure and calculate these properties. ${ }^{24-26}$ However, these studies use very high-frequency ultrasound (on the order of $\mathrm{MHz}$ ) and generally involve plate thickness resonances. None have shown that material properties may be extracted from a measurement of angular dependent sound transmission. This section proposes a method to determine the bending stiffness, $D$, of an isotropic plate using the angular dependent sound transmission setup described in Chapter 3.

Using the measurement process described above, it is possible to measure the coincidence angle by extracting the peak value of the transmission data at a specific frequency. As described by Eq. (2.6), this coincidence angle, $\theta_{C O}$, shifts from grazing incidence towards 
normal incidence as frequency is increased. Equation (2.6) can be solved for $\theta_{C O}$ as a function of frequency, yielding

$$
\theta_{C O}(f)=\sin ^{-1}\left[\left(\frac{c^{2}}{2 \pi f} \sqrt{\frac{m}{D}}\right)^{1 / 2}\right]
$$

Since $c$ is well defined and $m$ can be calculated using the geometry and mass of a bar, $D$ becomes the only unknown quantity in Eq. (D.1). By measuring the coincidence angle at various frequencies and curve-fitting this equation to the data, the bending stiffness can be measured.

The angular-dependent transmission through the aluminum bar was measured for frequencies between $20 \mathrm{kHz}$ and $70 \mathrm{kHz}$, in 1-kHz increments. Fig. D.1 shows a surface plot of frequency versus angle, where color on the surface plot denotes transmission levels. For the measurements below $35 \mathrm{kHz}$, a KEF Reference Series Hypertweeter was used. Between 35 and $50 \mathrm{kHz}$, the Parasonics 4012A piezoelectric source was used. Above $50 \mathrm{kHz}$, the Parasonics PAR58T piezoelectric source was used. Note that the coincidence angle shifts towards $0^{\circ}$ as frequency is increased.

The two angles at which coincidence occurred (the peaks mirrored about $0^{\circ}$ ) were extracted at each frequency interval. The coincidence angle at each frequency was calculated as the average of the two peaks. The results of these experiments, compared to the curve using known values of aluminum ( $E=70 \mathrm{GPa}, \rho=2700 \mathrm{~kg} / \mathrm{m}^{3}$, and $\sigma=0.33$ ) based on Eq. (D.1), are shown in Fig. D.2. 


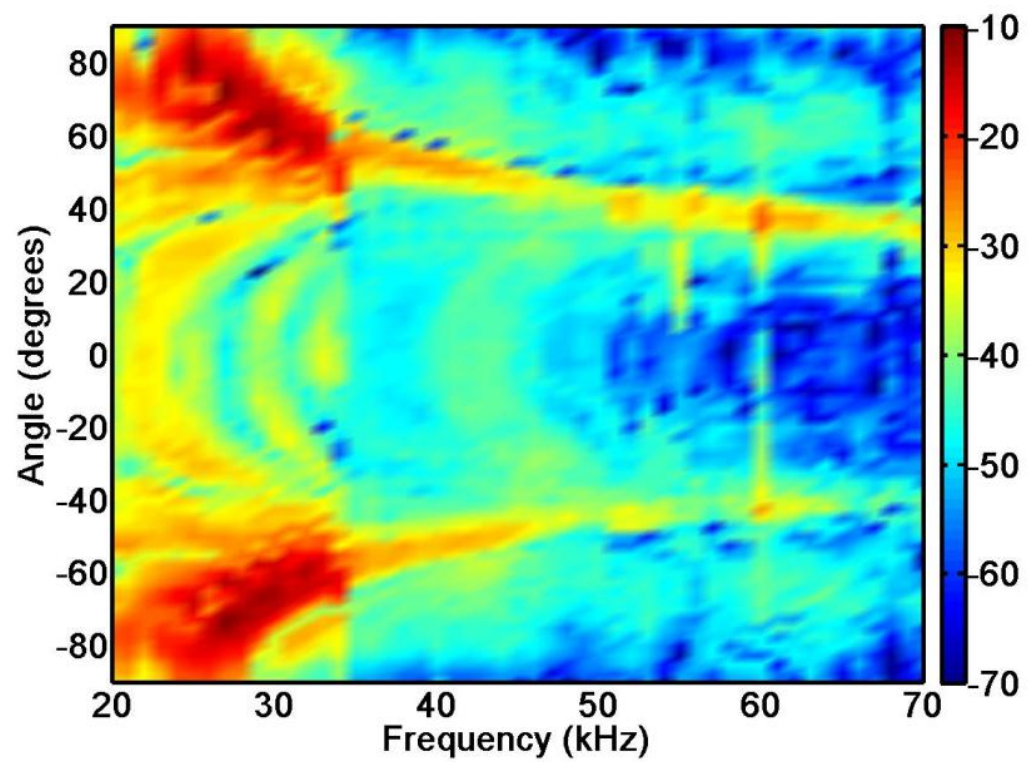

FIG. D.1. Surface plot of the transmission for frequency versus angle with color representing the transmission levels for the aluminum bar.

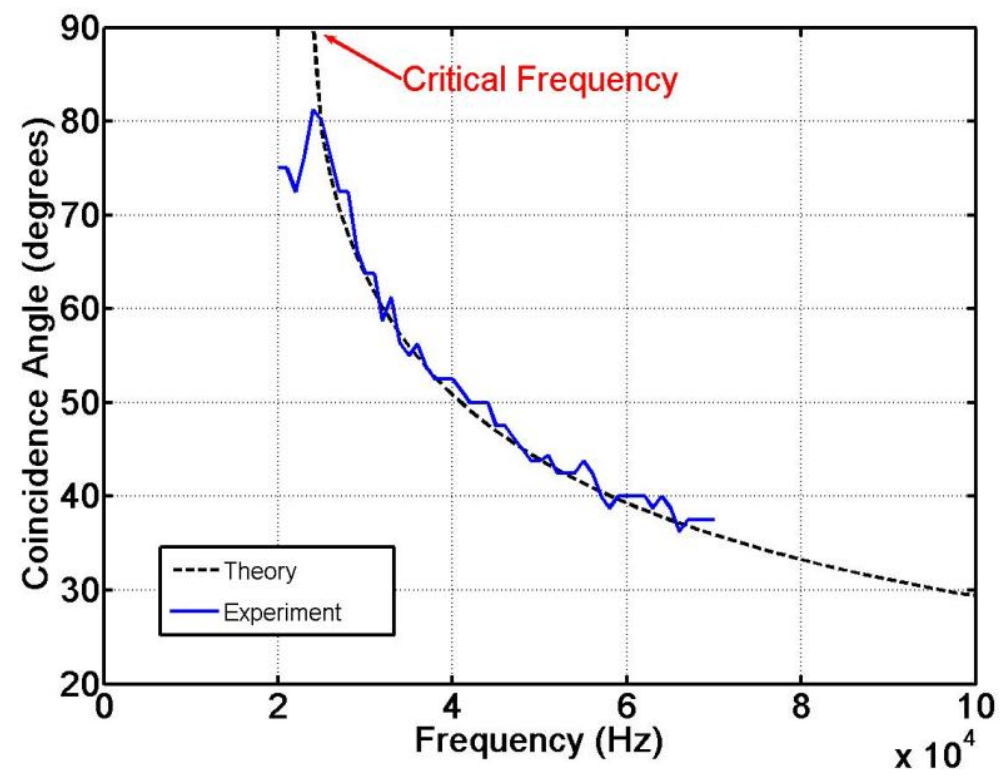

FIG. D.2. Coincidence angle versus frequency for the aluminum bar. 
Below the critical frequency $(24 \mathrm{kHz}$ for a $0.5-\mathrm{mm}$ aluminum bar), the peaks were indistinguishable because there should no longer be coincidence peaks present below the critical frequency. These data were therefore disregarded. Above the critical frequency, the coincidence angle decreases from $90^{\circ}$ towards normal incidence following the prescribed curve, with an average mean square error of $0.96^{\circ}$. Better angular resolution in the measurement system could potentially reduce this error further.

With these data, it is possible to fit the theoretical curve by minimizing the error using the least squares method. From this, the bending stiffness in Eq. (D.1) can be calculated. The data were curve-fitted in Mathematica using the NMinimize method of the FindFit function, the fit being based on a least squares approach. The experimental fitted value of the bending stiffness was 0.829 , while the calculated value of the bending stiffness, calculated using $E=70 \mathrm{GPa}$, $h=0.0005 \mathrm{~m}$, and $\sigma=0.33$ in Eq. (2.3), is 0.818 . This corresponds to a $1.3 \%$ error. This error could potentially be reduced further by increasing the angular resolution in the measurements, investigating the boundary conditions on the bar, or taking measurements at smaller frequency increments.

Using the experimental value for the bending stiffness, $0.005 \mathrm{~m}$ for the thickness and 0.33 for Poisson's ratio, Eq. (2.3) gives Young's modulus as $70.9 \mathrm{GPa}$, an error of $1.4 \%$ from the $70 \mathrm{GPa}$ used in the other calculations. This is actually closer to the value given by Kinsler et $a l .^{20}$ However, the aluminum bar is aluminum alloy 2024. Exact values for the Young's modulus for this bar were not measured, but various manufacturers give Young's moduli of 72.4 GPa or 73.1 GPa for 2024 alloys. Thus the fitted value of $70.9 \mathrm{GPa}$ may represent as much as 3\% error. The downside of this method is that a value for Poisson's ratio had to be assumed. 
Alternatively, Young's modulus could be measured using another method, such as by measuring longitudinal bar resonances, and a value of Poisson's ratio may then be extracted from the angular dependent sound transmission measurements. 